\title{
A Whole-Plant Monocot from the Early Cretaceous
}

\author{
Zhong-Jian Liu ${ }^{1,2}$, Li-Jun Chen ${ }^{2}$, Xin Wang ${ }^{3, *}$ \\ ${ }^{1}$ College of Landscape Architecture, Fujian Agriculture and Forestry University, \\ Fuzhou 350002, China \\ ${ }^{2}$ Shenzhen Key Laboratory for Orchid Conservation and Utilization, National Orchid \\ Conservation Center of China and Orchid Conservation \& Research Center of \\ Shenzhen, Shenzhen 518114, China \\ ${ }^{3}$ CAS Key Laboratory of Economic Stratigraphy and Palaeogeography, Nanjing \\ Institute of Geology and Palaeontology, CAS, Nanjing 210008, China
}

*To whom correspondence should be addressed. E-mail: xinwang@ @igpas.ac.cn

\begin{abstract}
The Yixian Formation (the Lower Cretaceous) of China is world famous for its fossils of early angiosperms. Although these diverse angiosperms demonstrate an unexpectedly great diversity, few are preserved as whole plants (not mention of monocots), making our understanding of them incomplete. Here, we report a fossil angiosperm, Sinoherba ningchenensis gen. et sp. nov (Sinoherbaceae fam. nov.), from the Yixian Formation of China; this fossil has a physically connected underground stem with fibrous rootlets, a stem with branches and nodes, leaves with parallel-reticulate veins, and a panicle of female flowers with an ovary surrounded by perianth. Morphological and phylogenetic analyses revealed that Sinoherba is an herbaceous monocot taxon. This newly discovered fossil underscores the great diversity of angiosperms in the Lower Cretaceous Yixian Formation.
\end{abstract}

Keywords: flower, fossil, Lower Cretaceous, China, angiosperms, herbaceous monocot. 
Although angiosperms constitute the most diversified plant group in the current world ${ }^{l}$, the origin, evolution and systematics of angiosperms are little understood or misunderstood. Early angiosperms including the famous Archaefructus ${ }^{1-4}$ have been reported from the Yixian Formation of northeastern China, but few of them are preserved as whole plants. Such fragmentary information hinders our full understanding of early angiosperms. To improve our understanding of early angiosperms, here, we report a new fossil plant, Sinoherba ningchengensis gen. et sp. nov. (Sinoherbaceae fam. nov.), from the Yixian Formation (the Early Cretaceous, 125 Ma). This fossil has a physically connected underground stem with nodes and fibrous rootlets; a stem with nodes, leaves and branches at the nodes; leaves with parallel-reticulate veins; a panicle arising from the axils of the leaves; and female flowers with an ovary surrounded by perianth. Morphological and phylogenetic analyses revealed that Sinoherba is an herbaceous monocot. Furthermore, Sinoherba demonstrates a novel character assemblage that helps elucidate the derivation of both gynoecia and growth habits of early angiosperms, underscores the great diversity of angiosperms in the Lower Cretaceous Yixian Formation, and suggests an earlier-than-assumed origin of angiosperms.

The Yixian Formation of China is well known for the Johel Biota as well as various fossil animals ${ }^{5-12}$ and plants ${ }^{13-17}$. Among the plants are Bryophyta, Lycopodiales, Equisetales, Filicales, Pteridospermae, Cycadales, Bennettitales, Ginkgoales, Czekanowskiales, Coniferales, and Angiospermae ${ }^{13-17}$. A general consensus exists with respect to the age of the Yixian Formation, namely, approximately $125 \mathrm{Ma}$ (the Barremian-Aptian, Lower Cretaceous) ${ }^{19}$. The new fossil plant was collected from an outcrop of the Yixian Formation in Dashuangmiao, Ningcheng, Inner Mongolia, China (Fig. 1). The specimen was preserved as a compression/impression in thin-layered siltstone, and embedded coalified residue was present. The specimen is $26 \mathrm{~cm}$ long and $5 \mathrm{~cm}$ wide; it is preserved on a slightly yellowish grey siltstone slab approximately $25 \mathrm{~cm} \times 38 \mathrm{~cm}$ (Fig. 2). The specimen was imaged using a Nikon D200 digital camera and a Nikon SMZ1500 stereomicroscope equipped with a Nikon DS-Fil digital camera. The distal portion of the plant was observed using a Leo 1530 VP scanning electron microscope (SEM) at Nanjing Institute of Geology and Palaeontology, CAS (NIGPAS). Sketches of the images were drawn using Photoshop 4.0 software.

\section{Angiospermae}

\section{Monocotyledoneae}

Family: Sinoherbaceae fam. nov.

Genus: Sinoherba gen. nov.

Type species: Sinoherba ningchengensis sp. nov.

Family and generic diagnosis: Herb, approximately $26 \mathrm{~cm}$ tall, with an underground stem. Nodes on the underground stem with lateral roots. Rootlets fibrous. Stem straight, with obvious nodes, with branches on the upper nodes. Leaves attached to the nodes in whorls, strap-like, with two orders of parallel veins and sparse meshes. Panicle arising from the axil of a leaf. Flowers dense on rachis, probably dioecious, pistil surrounded by 2-3 whorls of perianth. Ovary with a short distal style, partially 
separated in the distal half by a septum. Ovule basal, on the bottom of the ovary. Fruits ovate.

Etymology: Sino- Latin word for China, -herba from Latin word for herbaceous plant.

Species: Sinoherba ningchengensis sp. nov.

Specific diagnosis: the same as that of the family and genus.

Description: Whole plant including the roots, stem, leaves, and flowers, $258 \mathrm{~mm}$ long and $46 \mathrm{~mm}$ wide (Figs. 2-4). The underground stem is $3.4 \mathrm{~mm}$ in diameter and $39 \mathrm{~mm}$ in length, with several nodes (Fig. 3a). Fibrous roots are borne on the apex and nodes of underground stem, roots with 2-3 orders of fibrous rootlets (Figs. 3a, b). The stem is approximately $5 \mathrm{~mm}$ in diameter at the bottom, tapering to $1.4 \mathrm{~mm}$ distally, with evident and slightly swollen nodes (Figs. 2, 3c-e). The length of internodes varies from 7 to $27 \mathrm{~mm}$ (Figs. 2, 3c). Lateral branches/inflorescences and leaves are inserted on the nodes along the stem (Figs. 2, 3c-e, 4e). Younger lateral branches are in the axils of leaves (Figs. 2, 3e). The leaves are more concentrated on the basal half of the plant and are fully absent on two basal-most nodes of the stem (Figs. 2, 3c, f, g, 4e). A leafless node has leaf scars (Fig. 3d). The leaves are strap-shaped, up to $3.6 \mathrm{~mm}$ wide and $39 \mathrm{~mm}$ long, base contracted into a petiole (Fig. 3f) clasping the stem (Fig. $3 \mathrm{~g}$ ), apex acute to obtuse (Fig. 3h). The leaf veins are mostly parallel, with two orders of veins and elongated sparse meshes (Figs. 3f, i). The plant is mature, with two panicles (Figs. 4a, e). One of inflorescences arises from the apical node of the stem, the inflorescence has many young flowers, approximately $5 \mathrm{~cm}$ in length, with a bract at the base of the scape, mostly concentrated in the distal portion of the plant (Figs. 4a, b). The bract of inflorescence is oblong-lanceolate, apex obtuse (Fig. 4c). The flowers are terminal, alternately arranged along the peduncle, with a superior ovary, $0.2 \mathrm{~mm}$ wide and $0.2 \mathrm{~mm}$ long, surrounded by perianths (foliar parts) (Figs. 2, 4a, b). Another inflorescence, whose scape arises in the axil of a leaf on the stem, approximately 3.5 $\mathrm{cm}$ in length (Fig. 4a). The inflorescence is approximately $3 \mathrm{~cm}$ long, with several flowers/fruits (Fig. 4e). The flowers (fruits) are densely arranged along the peduncle and have 2-3 layers of perianth, $6 \mathrm{~mm}$ long and $5 \mathrm{~mm}$ wide, pedicel short, approximately $1 \mathrm{~mm}$ long (Figs. 4e, f). No male parts are recognized. The gynoecium includes a conical ovary and a distal style and is surrounded by perianth (Figs. $4 \mathrm{f}-\mathrm{h}$ ). The ovary is unilocular proximally but bilocular distally, with a wall approximately 67 $\mu \mathrm{m}$ thick and a basal ovule (Figs. 4d, g, h). The style is stout, cylindrical, up to 372 $\mu \mathrm{m}$ in length and $155 \mu \mathrm{m}$ in diameter (Fig. $4 \mathrm{~h}$ ). The ovule is basal, approximately 670-1000 $\mu \mathrm{m}$ wide and 460-590 $\mu \mathrm{m}$ high (Fig. 4g, h, 6c-d).

Etymology: ningcheng-, for Ningcheng City, Inner Mongolia, the fossil locality.

Holotype: LJNG0002 a \& b (Fig. 2).

Type locality: Liujianangou, Dashuangmiao Town, Ningcheng, Inner Mongolia, China $\left(41^{\circ} 30.3792^{\prime} \mathrm{N}, 118^{\circ} 51.2465^{\prime} \mathrm{E}\right.$, Fig. 1$)$.

Stratigraphic horizon: the Yixian Formation, equivalent to the Barremian-Aptian, Lower Cretaceous (125 Ma).

Depository: The Orchid Conservation \& Research Center of Shenzhen, China (NOCC). 
The small size and maturity (suggested by the presence of flowers) of Sinoherba indicate that it is an herbaceous plant. To the best of our knowledge, herbaceous growth habit among living seed plants is restricted to angiosperms. Therefore, the herbaceous growth habit of Sinoherba per se suggests that this plant is likely an angiosperm $^{18}$, a conclusion supported by other features of Sinoherba.

Sinoherba has a panicle subtended by an inflorescence bract, and the flower is composed of a pedicel, perianths and a gynoecium. Importantly, via their enclosed ovule before pollination, angiosperms are easily distinguished from gymnosperms; enclosed ovules before pollination have never been reported in gymnosperms ${ }^{19,20}$. As seen in Figs. 4d, $\mathrm{f}-\mathrm{h}$ and $6 \mathrm{c}-\mathrm{d}$, the basal ovule is fully enclosed by an ovary, the latter of which is completely sealed at the tip by a short style and is partially bilocular distally (Figs. 4g, h). These features are characteristic of angiosperms. As such, the angiospermous affinity of Sinoherba suggested above is confirmed.

The leaves of Sinoherba are arranged at its nodes, and the leaf petioles clasp the stem (Figs. 2, 3c-i); the leaves are not spirally arranged as might be expected according to angiosperm evolution theories ${ }^{21}$. Spiral phyllotaxy was formerly assumed to be ancestral in angiosperms ${ }^{21}$, and unfortunately, this belief is still pervasive among botanists. However, the phyllotaxy of Sinoherba apparently contradicts this belief. Sinoherba presents leaf characteristics that suggest a monocot affinity. The leaves of Sinoherba are oblong-lanceolate and with reticulate veins. The veins are of two orders: the first-order parallel veins converge at the apex, and the second-order veins join the first-order ones (Figs. 3f, h, i). The venation of Sinoherba is approximately parallel, a venation rarely seen in eudicots. The underground stem with fibrous rootlets (Fig. 3a) excludes a possible Eudicot affinity for Sinoherba, as this type of stem morphology is frequently seen in monocots but rarely seen in eudicots $^{22}$. Such a character assemblage and herbaceous habit suggest that Sinoherba is a monocot.

Sinoherba is probably an helobious plant. An herbaceous nature and roots borne on the basal nodes of underground stem suggest an helobious habitat. The absence of leaves and the presence of leaf scars on the basal nodes of the stem in Sinoherba (Figs. $3 c, d)$ suggest that the leaves must have fallen off before fossilization. Sinoherba has two types of inflorescences: one is a developing panicle, and the other appears to be an infructescence that probably formed during a different flowering season. In contrast to the presence of leaves on the nodes discussed above, this phenomenon suggests that Sinoherba is likely a perennial and deciduous herb that can adapt to environment changes (suggested by leaf falling). Similar character assemblage has been reported for various monocot families, e.g., the Helobiae ${ }^{23}$.

To decipher the origin, evolution and systematics of angiosperms correctly and confidently, all hypotheses must be based on fossil evidence. Various fossils of early angiosperms from the Yixian Formation, including those of Archaefructus ${ }^{1-4}$ and Sinocarpus $^{24,25}$, have shed important light on these issues, but few of those fossils are of whole plants. The fossil of Sinoherba is unique because it is an herbaceous whole plant, including the roots, stem, leaves, and inflorescences. Phylogenetic analyses of the morphological characters as well as the combination of characters and molecular 
data of both extant plants and Sinoherba demonstrate that Sinoherba is a monocot taxon $^{27}$ (Figs. 5, S1, S2).

When all the morphological characters of Sinoherba are evaluated in a phylogenetic context, Sinoherba nests in the angiosperm clade. The angiosperm clade is divided into three subclades: Archaefructus, monocots and eudicots. Archaefructus is a basal clade and is the sister of the monocots and eudicots. The Amborellaceae is the most basal subclade among the eudicots, while the Nymphaeaceae is the most basal subclade among monocots. Sinoherba is a monocot taxon and is the sister of the Najadaceae, an aquatic plant family (Fig. S1). Phylogenetic relationships were reconstructed using a combination of morphological characters and plastid DNA regions. The phylogenetic relationships revealed in this analysis (Fig. 5, Fig. S2) are similar to those revealed via morphologic characters alone. All analyses consistently placed Sinoherba among the monocots and indicated that it represents a sister of the extant Araceae and Helobiae families (Najadaceae, Alismataceae and Hydrocharitaceae). We hope future studies can test whether Sinoherba stands for the basalmost monocot. The characters unique to the Sinoherbaceae are illustrated in reconstruction of $S$. ningchengensis (Fig. 6).

The occurrence of basal ovule in S. ningchengensis is unexpected for classical angiosperm evolution. However, similar way of gynoecium forming is commonly seen in angiosperm and is recently termed as mixomery ${ }^{26}$. Apparently, this new findings in extant as well as fossil angiosperms deserve attention in future study of angiosperm evolution.

The discovery of $S$. ningchengensis and the phylogenetic and morphological analyses refute the Early Cretaceous origin of monocots. Instead, our results favour a more ancient origin of angiosperms.

\section{References and Notes}

1 Sun, G., Dilcher, D. L., Zheng, S. \& Zhou, Z. In search of the first flower: a Jurassic angiosperm, Archaefructus, from Northeast China. Science 282, 1692-1695, (1998).

2 Sun, G. et al. Archaefructaceae, a new basal angiosperm family. Science 296, 899-904, (2002).

3 Ji, Q., Li, H., Bowe, M., Liu, Y. \& Taylor, D. W. Early Cretaceous Archaefructus eoflora sp. nov. with bisexual flowers from Beipiao, Western Liaoning, China. Acta Geologica Sinica 78, 883-896, (2004).

4 Wang, X. \& Zheng, X.-T. Reconsiderations on two characters of early angiosperm Archaefructus. Palaeoworld 21, 193-201, (2012).

5 Teng, F., Lü, J., Wei, X., Hsiao, Y. \& Pittman, M. New material of Zhenyuanopterus (Pterosauria) from the Early Cretaceous Yixian Formation of western Liaoning. Acta Geologica Sinica (English edition) 88, 1-5, (2014).

6 Wang, W. et al. Mesozoic stratigraphy and paleontology in western Liaoning. Geological Publisher, Beijing, 1989.

7 Wang, W.-L. et al. The age of the Yixianian stage and the boundary of Jurassic-Cretaceous, --- the establishment and study of stratotype of the 
Yixianian stage. Geological Review 51, 234-242, (2005).

8 Liu, Y., Sinitshenkova, N. D. \& Ren, D. A new genus and species of stonefly (Insecta: Plecoptera) from the Yixian Formation, Liaoning Province, China. Cretaceous Research 28, 322-326, (2007).

9 Yao, Y., Cai, W. \& Ren, D. Fossil flower bugs (Heteroptera: Cimicomorpha: Cimicoidea) from the Late Jurassic of Northeast China, including a new family, Vetanthocoridae. Zootaxa 1360, 1-40, (2006).

10 Ren, D. Flower-associated Brachycera flies as fossil evidences for Jurassic angiosperm origins. Science 280, 85-88, (1998).

11 Ren, D., Shih, C., Gao, T., Yao, Y. \& Zhao, Y. Silent stories. Science Press, Beijing, 2010.

12 Gao, T. et al. New transitional fleas from China highlighting diversity of Early Cretaceous ectoparasitic insects. Current Biology 23, 1261-1266, (2013).

13 Wu, S.-Q. A preliminary study of the Jehol flora from the western Liaoning. Palaeoworld 11, 7-57, (1999).

14 Sun, G., Zheng, S., Dilcher, D., Wang, Y. \& Mei, S. Early angiosperms and their associated plants from Western Liaoning, China. Shanghai Technology \& Education Press, Shanghai, 2001.

15 Zheng, S., Li, N., Li, Y., Zhang, W. \& Bian, X. A new genus of fossil cycads Yixianophyllum gen. nov. from the Late Jurassic Yixian Formation, Western Liaoning, China. Acta Geologica Sinica 79, 582-592, (2005).

16 Dong, C., Yang, X. \& Zhou, Z. in Daohugou Biota (ed Diying Huang) 252-302 (Shanghai Science and Technology, 2016).

17 Li, G. \& Luo, Z. X. A Cretaceous symmetrodont therian with some monotreme-like postcranial features. Nature 439, 195-200, (2006).

18 Friis, E. M., Crane, P. R. \& Pedersen, K. R. The early flowers and angiosperm evolution. Cambridge University Press, Cambridge, 2011.

19 Tomlinson, P. B. \& Takaso, T. Seed cone structure in conifers in relation to development and pollination: a biological approach. Canadian Journal of Botany 80, 1250-1273, (2002).

20 Wang, X. The dawn angiosperms. Springer, Heidelberg, 2018.

21 Cronquist, A. The evolution and classification of flowering plants. New York Botanical Garden, Bronx, 1988.

22 Judd, W. S., Campbell, S. C., Kellogg, E. A. \& Stevens, P. F. Plant systematics: a phylogenetic approach. Sinauer Associate Inc., Sunderland, MA, 1999.

23 Sun, X. in Flora Reipublicae POPULARIS sINICAE Vol. 8 32-190 (1992).

24 Leng, Q. \& Friis, E. M. Sinocarpus decussatus gen. et sp. nov., a new angiosperm with basally syncarpous fruits from the Yixian Formation of Northeast China. Plant Systematics and Evolution 241, 77-88, (2003).

25 Leng, Q. \& Friis, E. M. Angiosperm leaves associated with Sinocarpus infructescences from the Yixian Formation (Mid-Early Cretaceous) of NE China. Plant Systematics and Evolution 262, 173-187, (2006).

26 Sokoloff, D. D., Nuraliev, M. S., Oskolski, A. A. \& Remizowa, M. V. 
Gynoecium evolution in Angiosperms: Monomery, pseudomononomery, and mixomery. Moscow University Biological Sciences Bulletin 72, 97-108, (2017).

27. To determine the phylogenetic position of Sinoherba, we sampled 169 angiosperm taxa. A morphological matrix was constructed de novo (Table S1); the matrix integrated the majority of the morphological features coded (Table S2) by Moreau et al. ${ }^{28}$. For a broad phylogenetic analysis, a total of 141 taxa were included in the analysis of the data set comprising four plastid DNA (rbcL, matK, trnL-F and $p s b A-t r n H$ ) sequences and one nuclear (ITS) sequence (The GenBank accession numbers are listed in Table S3). The DNA sequences were aligned with MEGA $5.05^{29}$ using the Muscle model ${ }^{30}$ and manually adjusted to account for obvious or missing inserts. We translated the basic DNA sequence groups A, T, G, and C into 1, 2, 3, and 4, respectively, and subsequently combined the DNA sequences and score information of the morphological characteristics. The phylogenetic analyses were carried out using the maximum parsimony (MP) method. The MP analysis was carried out using PAUP $4.0 \mathrm{~b} 10^{31}$.

28. J.-D. Moreau et al. Verneda hermaphroditica gen. \& sp. nov.: A new flower head from the early Late Cretaceous (middle Cenomanian) of southeastern France. Taxon 65(4): 823-838 (2016).

29. K. Tamura et al. MEGA5: molecular evolutionary genetics analysis using maximum likelihood, evolutionary distance, and Maximum Parsimony methods. Molecular Biology and Evolution 28 (10): 2731 (2011).

30. R. C. Edgar. Muscle: multiple sequence alignment with high accuracy and high throughput. Nucleic Acids Research 32 (5), 1792-1797(2004).

31. D. L. Swofford. PAUP*. Phylogenetic analysis using parsimony (*and other methods), v. 4.0 beta 10 (Sinauer, Sunderland, 2003).

32. We thank Ms. Chunzhao Wang for her assistance with the SEM. This research was supported by the National Natural Science Foundation of China (41688103, 91514302); the Chinese Academy of Sciences (Grant No. XDPB05) via a grant awarded to X. W.; the State Forestry Administration of China (No. 2005-122); the Science and Technology Project of Guangdong (No. 2011B060400011); and the Special Funds for Environmental Projects of Shenzhen (No. 2013-02), which was awarded to Z.-J. L. 


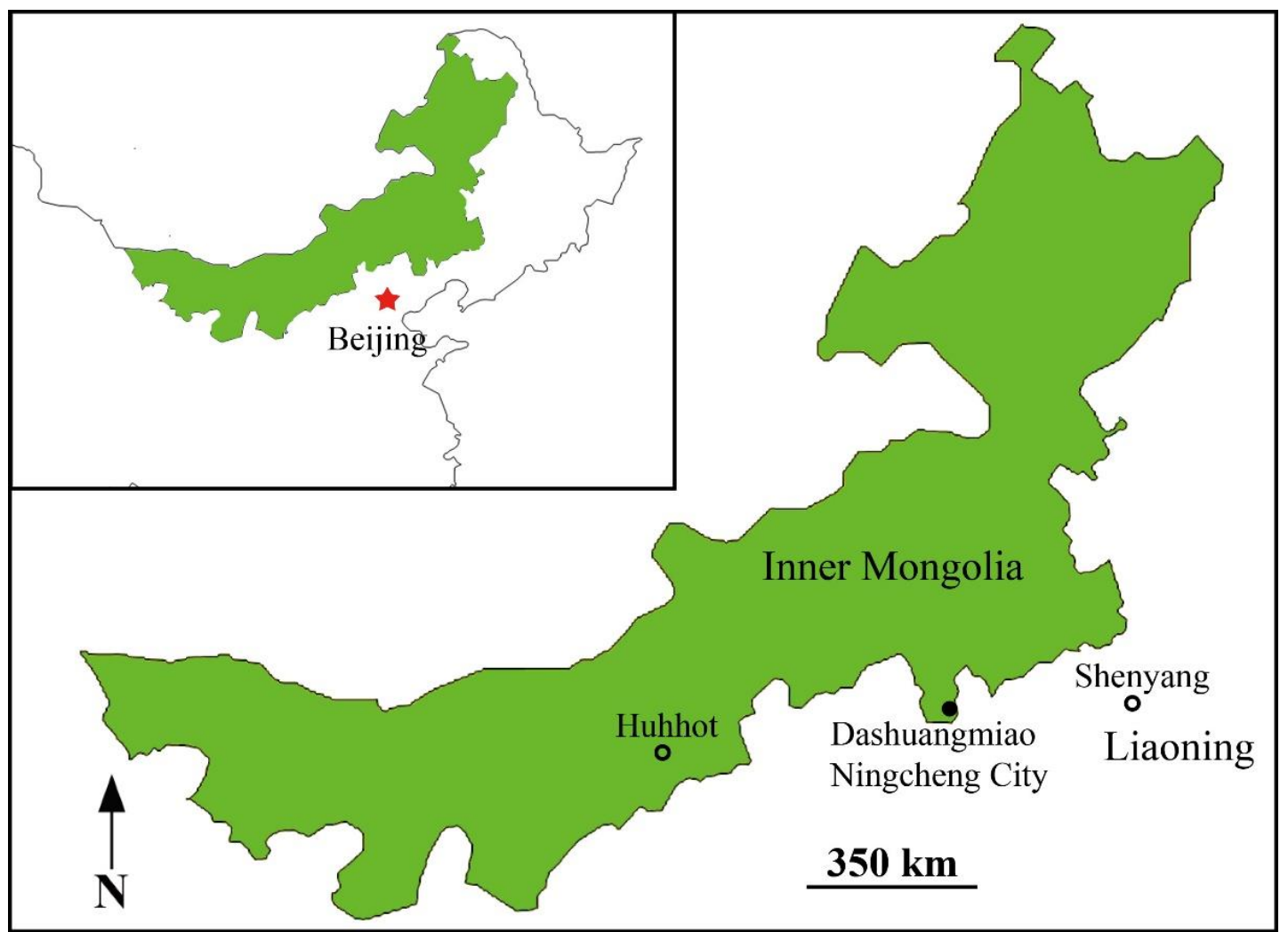

Figure 1. Fossil locations. The map shows the Dashuangmiao locality in Ningcheng of Inner Mongolia from where the fossil Sinoherba ningchengensis gen. et sp. nov. was collected. The inset map shows the location of Inner Mongolia in northeastern China (the asterisk indicates Beijing). 

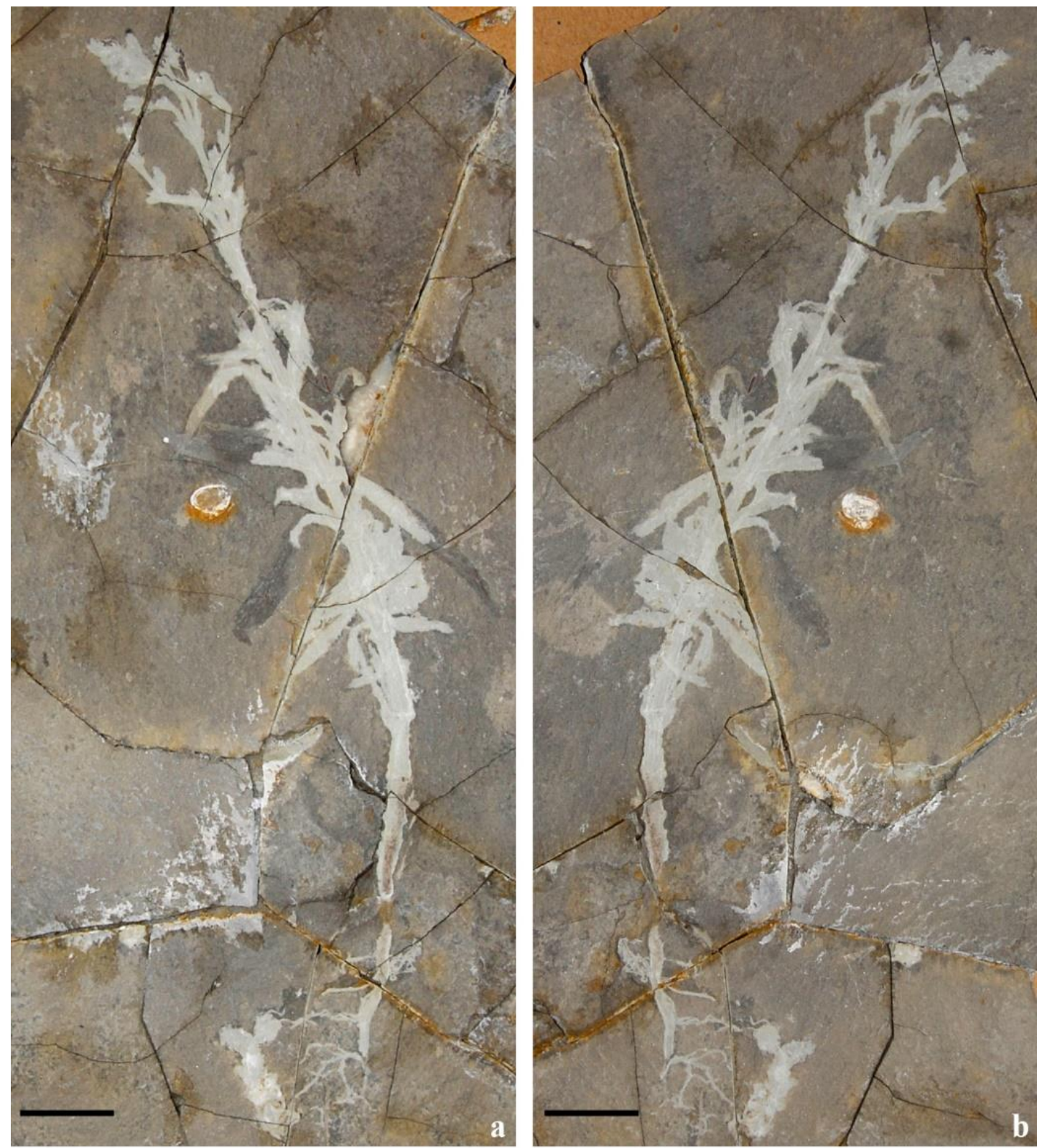

Figure 2. General view of the holotype of Sinoherba gen. nov. The whole plant includes the rootlets, stem, leaves and inflorescences. Bar $=2 \mathrm{~cm}$. 

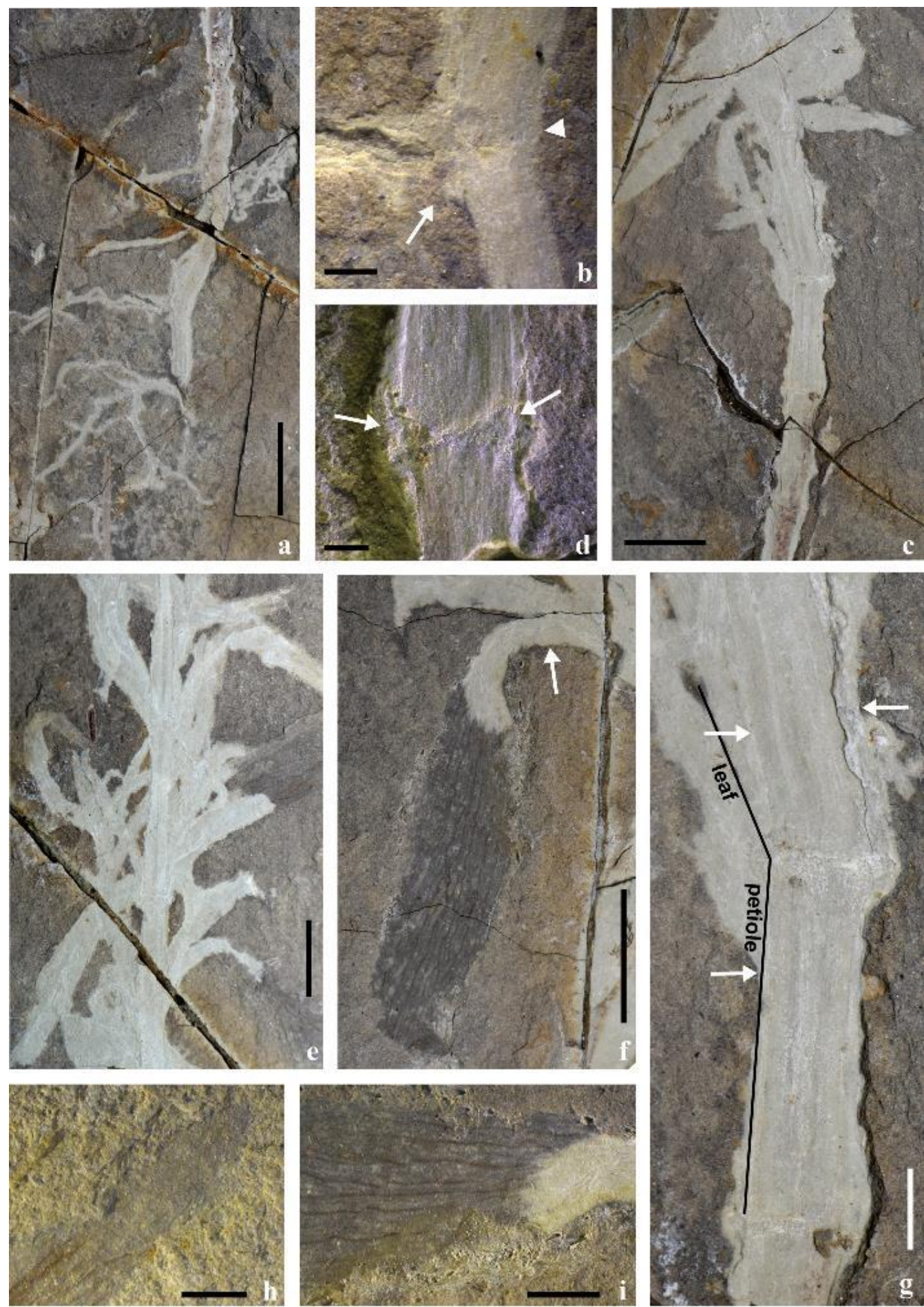

Figure 3. The vegetative organs of Sinoherba gen. nov. a. Fibrous roots are borne on the apex and nodes of an underground stem. Bar $=1 \mathrm{~cm}$. $\mathbf{b}$. A root (arrow) is borne on a node (arrowhead). Bar $=1 \mathrm{~mm}$. c. A stem with swollen nodes and some nodes without leaves. Bar $=1 \mathrm{~cm}$. d. A node with leaf scars (arrows). Bar $=1 \mathrm{~mm}$. e. The stem together with a branch at the node. Bar $=1 \mathrm{~cm}$. f. A leaf with parallel venation attached to a node. Note the leaf base that is contracted into the petiole (arrow). Bar = $1 \mathrm{~cm}$. g. The petiole of a leaf clasping the stem (arrows). Bar $=3 \mathrm{~mm}$. h. The apex of a leaf. Bar $=1 \mathrm{~mm}$. i. Detailed view of the venation of the leaf shown in (f). Bar $=2$ $\mathrm{mm}$. 

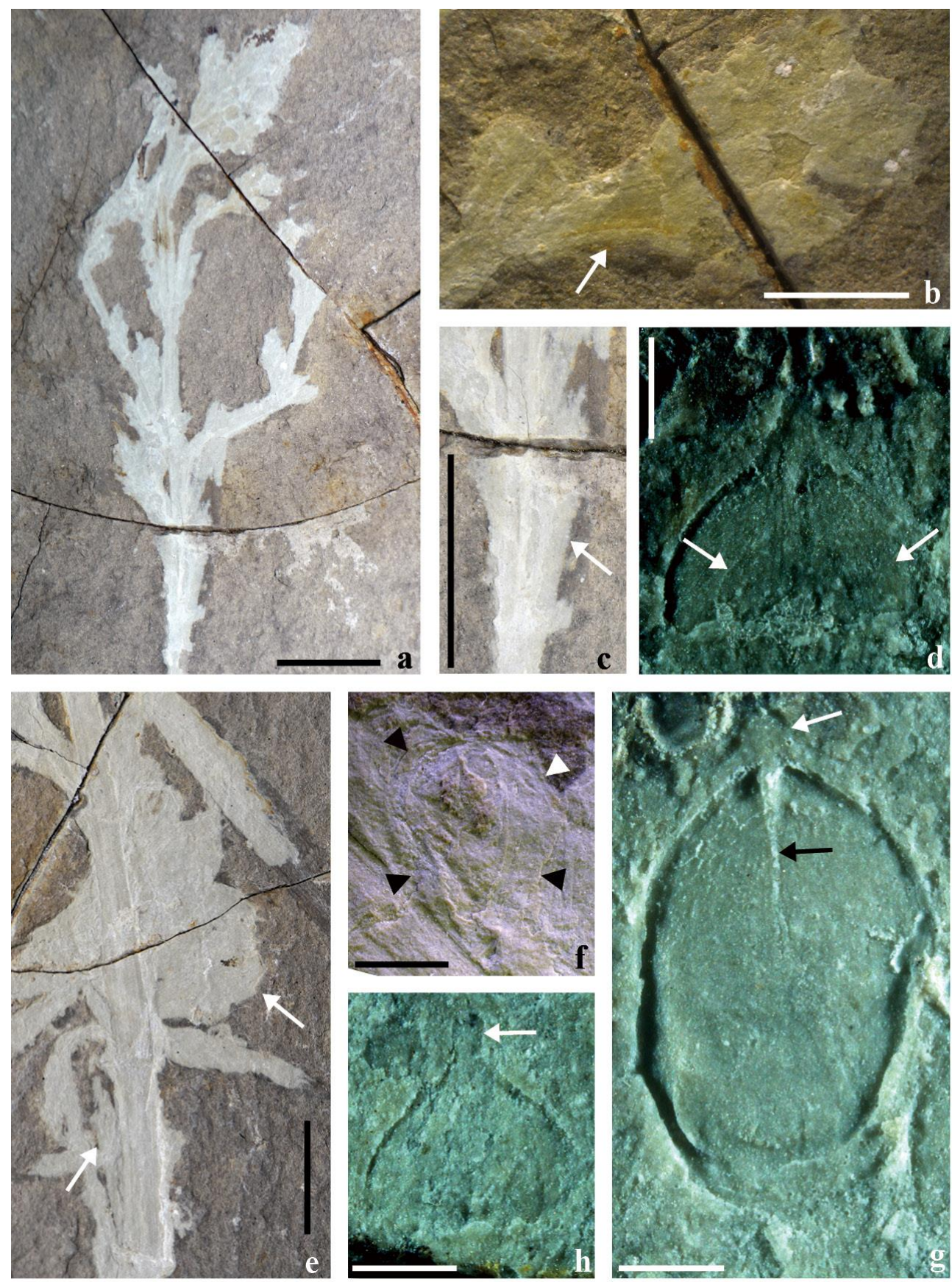

Figure 4. The reproductive organs of Sinoherba gen. nov. a. A panicle with many flowers. Bar $=1 \mathrm{~cm}$. b. Flowers borne on a pedicel (arrow). Bar $=2 \mathrm{~mm}$. c. A bract at the base of an inflorescence (arrow). Bar $=1 \mathrm{~cm}$. d. An ovary with basal ovule (arrows). Bar $=0.5 \mathrm{~mm}$. e. An inflorescence arisen from the axils of a leaf (arrows). Bar $=1 \mathrm{~cm}$. f. A flower of the inflorescence from the axil of a leaf on the stem in Fig. 4e, showing the ovary surrounded by approximately three layers of perianths (arrowheads). Bar $=2 \mathrm{~mm}$. g. An ovary split through its centre, showing its style (white arrow) and half septum (black arrow). Bar $=0.5 \mathrm{~mm}$. h. Detailed view of the distal portion of an ovary, showing the distal style (arrow) on the ovary. Bar $=0.5 \mathrm{~mm}$. 


\section{eudicots}

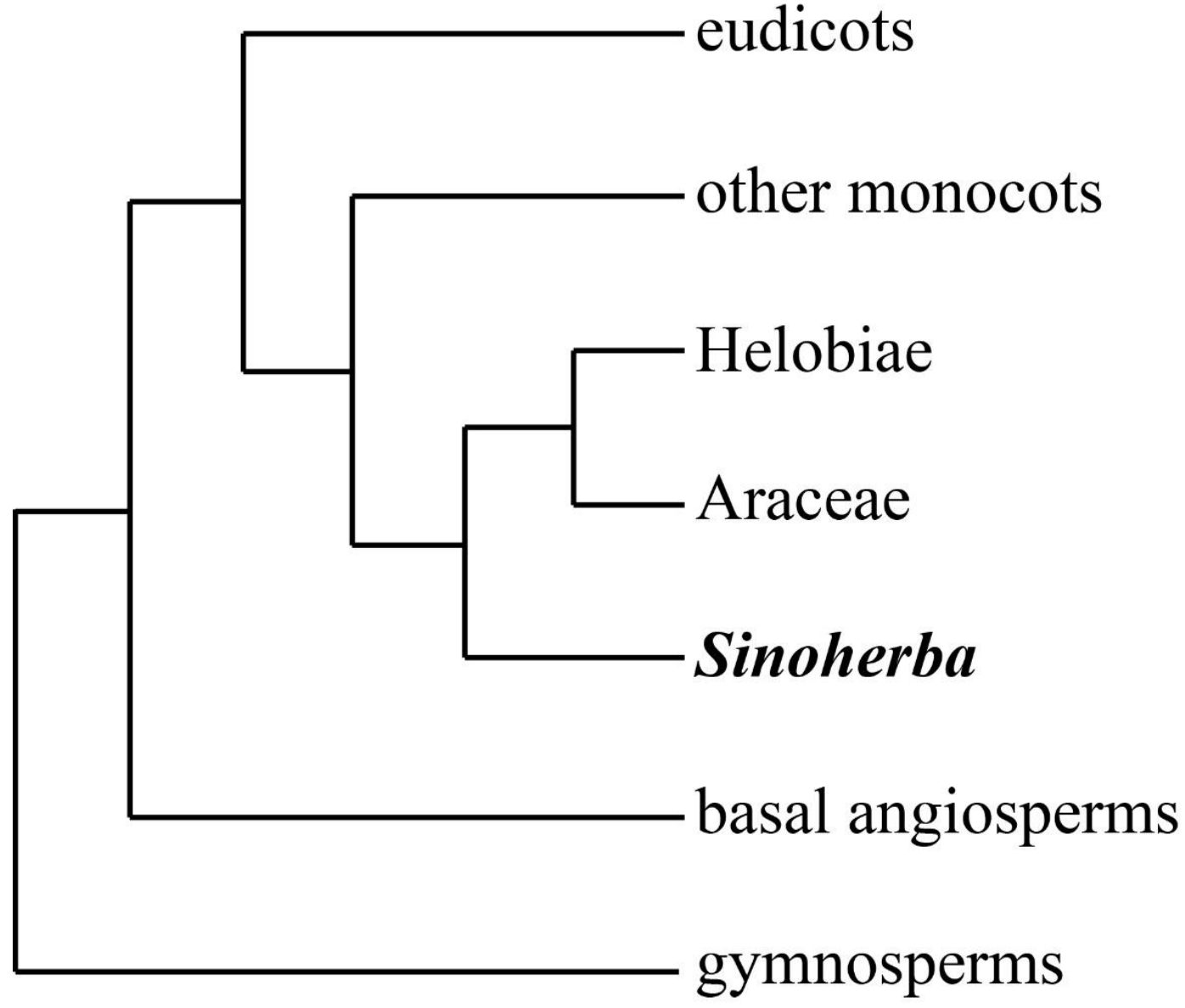

Figure 5. A phylogenetic tree (simplified) was reconstructed using a combination of morphological characters and plastid DNA regions (see details in Supplementary Fig. 2). 


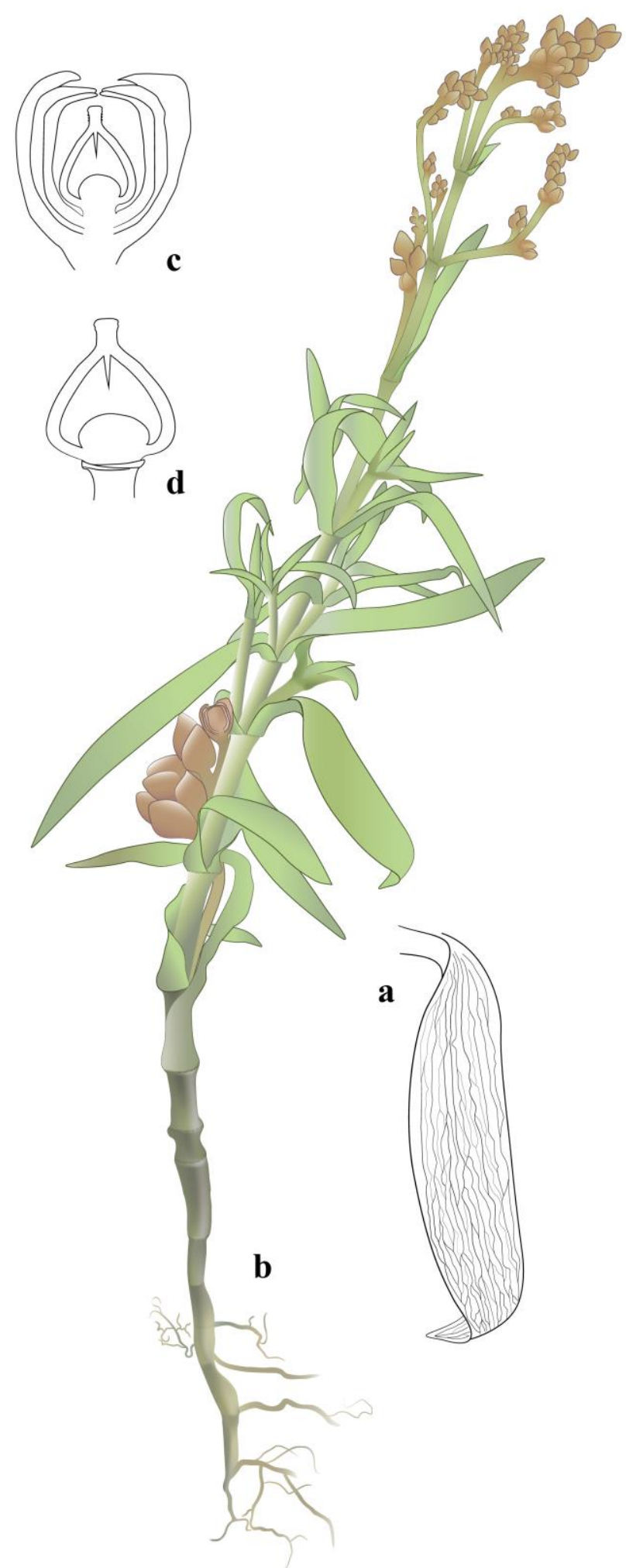

Figure 6. Reconstruction of Sinoherba ningchengensis gen et sp. nov. The plant is herbaceous and is composed of an underground stem with fibrous rootlets borne on the nodes, a stem with leaves (a) and branches in axils of bracts borne on the nodes, and inflorescences that both protrude from the axils of the leaves and are borne on the terminal stem (b). The pistil is surrounded by perianth (c) and the ovule on the bottom of the ovary (d). The colour of the flower was artificially created. 


\section{Supplementary Information}

Table S1. Taxa and value information.

\begin{tabular}{|c|c|c|}
\hline No. & Taxa & Characters \\
\hline 1 & Vojnovskyales & $1 ? 1 ? 16 ? ? ? 0 ? ? ? 353011 ? 1103000340600 ? ? 10110 ? 1400200 ? ? 032 ? ? ? ? 0 ? ? ? ? ? 74042 ? 705105 ? 000150300 ? 0100 ?$ \\
\hline 2 & Caytoniales & $1 ? 1 ? 1 ? ? ? ? 1 ? 0 ? 3530 ? 1 ? 110210 ? 030111 ? 01211102400200010320500000 ? 31040423501103 ? 000121100 ? 0100 ?$ \\
\hline 3 & Corystospermales & (1 2)?1?160??1?0(0 3)353011?11?01??03(0 1)5(0 1)02(0 3)1211102400200010320500000?31040423501103?000111200?0100? \\
\hline 4 & Peltaspermales & $1 ? 1 ? 130 ? ? 0 ? 0 ? 353011 ? 11 ? 01 ? ? 030(134)(0$ 1)0(0 2)(0 1)1211102400200010320202000?31740423501105?000110310?0100? \\
\hline 5 & Glossopteridales & $1 ? 1 ? 1601 ? 0(12) 0(0$ 3)353011?110(2 3)0?00305(0 1)10012111024002001103201(0 2)(0 1)000?3054042350(0 1)10500011(0 2)1(3 4)(0 1)0000002 \\
\hline 6 & Pentoxylales & (1 3)?1?1(6 7)015110(0 3)353011?110300?0307110(0 1)121110101020?0103204?2000?31740423505005?000150300?0100? \\
\hline 7 & Utrechtiaceaee & 1?10160??100?353011?11(0 1)(2 3)000440(3 8 B)102(0 3)1111100400200122301010000?3174042350511510001(1 2)030110100? \\
\hline 8 & Ullmanniaceae & 1?10?60???0??353011?110???????(5 9)??2?121110?40020012?3202?1??0?3174042350511510?012030??0000? \\
\hline 9 & Stachyotaxus & 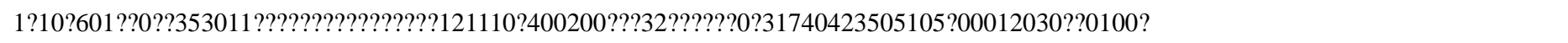 \\
\hline 10 & Palissyaceae & 1?10?60???0??353011??????????????12111??400200???32??????0?31740423505105?0?012030??0100? \\
\hline 11 & Emporiaceae & $1 ? 1016015000(0$ 3)353011011(0 1)3000??????2(0 3)1?101024002001123201?1000???74042350515????01??????????0 \\
\hline 12 & Thucydiaceae & $1 ? 101601 ? ? 00 ? 35301101103000540(3$ 8)0010111010?4002001223205?1000?31740423505105?0?010030??0000? \\
\hline 13 & Trichopitys & $1 ? 101 ? 01 ? 0 ? ? ? 3530 ? 1 ? 11030004 ? 0400 ? ? 12 ? 01(0$ 1)040020012232??02??0?317404235051?5?00015??00?0000? \\
\hline 14 & Cordaitales & $101016015000(0$ 3)353011?1103000(3 4)40(3 7)00(0 2)11?11(0 1)02400200?22320201000?31740423505005100010030000100? \\
\hline 15 & Lyginopteris & 2?1016(0 1)1?0101353011111?(0 3)100(0 4)104002?12?1100400200022320202000931740423505105001010100000000? \\
\hline 16 & Medullosans & (1 2)?101711?0101353011?110(0 3)1000101100012?110240020002232020200093174042350520500001(0 1)030000000? \\
\hline 17 & Primocycas & 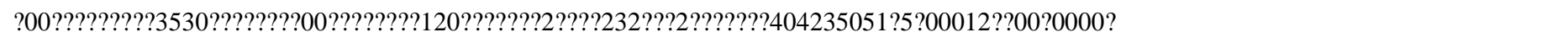 \\
\hline 18 & Cycadaceae & $1 ? 00171 ? ? 0(0$ 1)01353010?110310044(0 1)5100?120111(0 1)400200012320202000?3174042350510500001(2 3)0300000002 \\
\hline 19 & Zamia & 1?00171??00?13530?0??103100???????120???????2??0123202?20?0?317404235051?5000013??00?00002 \\
\hline 20 & Encephalartos & 1?00171??00?13530?0??103100????????120???????2??0123202?20?0?317404235051?5000013??00?00002 \\
\hline
\end{tabular}




\begin{tabular}{|c|c|c|}
\hline 21 & Yimaia & 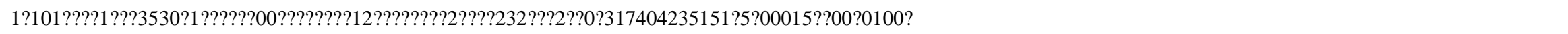 \\
\hline 22 & Ginkgo & $1110160 ? ? 10 ?(0$ 3)353011??103?00??0????0121???????2??012320502000?317404235151?5000010??000(0 1)100(1 2) \\
\hline 23 & Ferugliocladaceae & $1 ? 10 ? 60 ? ? ? 0 ? ? 353011 ? 11030 ? ? ? 40(3 \mathrm{C}) 10 ? ? 121110 ? 400200 ? ? ? 32 ? ? ? 1 ? ? 0 ? 31740423505105 ? 0 ? 012030 ? ? 0000 ?$ \\
\hline 24 & Majonicaceae & 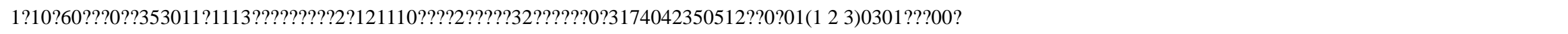 \\
\hline 25 & Cephalotaxaceae & $1 ? 1016015 ? 00 ? 353011 ? 110\left(\begin{array}{l}1 \\
2\end{array}\right) 00 ? 5403100 ? 121110 ? 40020012 ? 3200 ? 2000 ? 317404235051051000120301001002$ \\
\hline 26 & Schizolepis & 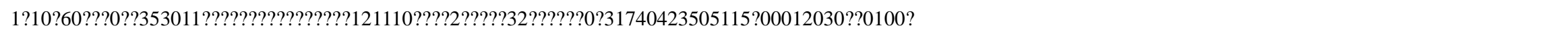 \\
\hline 27 & Pinaceae & $1 ? 1016015100(0$ 3)3530111110(0 1 2)00?540(3 B)102?1(1 2)1110140020012?3202?(0 14 5)000?31740423505115100012030100100(0 12$)$ \\
\hline 28 & Taxodiaceae & $1 ? 1016015000 ? 353011 ? 110300 ? 540(13) 10(0$ 2)?121110?40020002?3202?2000?3174042350510010?0120301100000 \\
\hline 29 & Cupressaceae & 1?1016015000?353011?111(1 2 4)00?5408?0(0 2)?1(1 2)1110?40020011?3202?2000?31740423505105100012030100100(0 2) \\
\hline 30 & Podocarpaceae & (1 3)?1016015000(0 3)353011?110(1 2)00?540(3 9)1020121110(0 1)4002001103202?(0 14 5)000?317404235051151000120301001002 \\
\hline 31 & Araucariaceae & $1 ? 1116015000 ? 3530110110(2$ 3)00?(3 4)40(5 9)10??1(1 2)111014002001103202?2000?317404235051151000120301001001 \\
\hline 32 & Bennettitales & 1?1?160??000(0 3)353011?110310?0?(0 1)1(0 1)(0 1)?11(0 2)11(0 1)000002000103201020?0?3174042150510??000150310?01002 \\
\hline 33 & Ephedra & (1 3)?11160???0112?1111?110(2 4)00?340800??12111102002000103205?200(0 1)???740421505105100010?010010002 \\
\hline 34 & Gnetum & (1 2)?111(3 6)????0111?1111?110400?0?0911??12111102002000103205?201????740421505005101010?010110002 \\
\hline 35 & Welwitschia & 3?111(3 6)????0111??111?110400????7?1001(0 2)111104002000103205?200????740421505105101010?010100002 \\
\hline 36 & Archaefructus & 0?1?0?22?0??????????11010??440400??1011001400200010?001?110????00102030(0 1)?01?00112030????00? \\
\hline 37 & Nymphaeaceae & 02110A22(2 3)01020030?13110300(0 1)6?0D01101011001100000010000502(0 2)????10(0 2)02313?00(0 1)4000115030101100(2 3) \\
\hline 38 & Hydatellaceae & 04110?22?0?1?0??1?1?1100000740300?01111011400000010?00502(0 2)????1500031B1200(0 6)100112030111000? \\
\hline 39 & Cyclanthaceae & (0 2 3)?1106?2???1?0??1?1?1100(0 1)0?(6 7)?040??211111112(0 1)00100101005?20?????2(2 3)0331(3 B)10?1310011(2 5)0301011003 \\
\hline 40 & Illiciaceae & (1 3)0111601?0?1?01?1?1111000000?(0 1)9110(0 1)10110(0 1)10000000(0 1)0(0 2)0050200100?0000400020101001150301111002 \\
\hline 41 & Sinoherba & 0?1?0?22?0??????????110200?340(35)01??12?111?????0????????????????500331202????0?11003???????? \\
\hline 42 & Magnoliaceae & (1 3)(2 3 5)111601?0011(0 2)11111(0 1)11030010?09110(0 1)1011001000000010(0 1 2)00502001(3 6 7)4(0 1)00002001101(0 3 7)1001150301111002 \\
\hline 43 & Amborellaceae & (1 3)0111601?000?353011?110(0 3)0000?(0 1)9110(0 1)12111(0 1)100(0 1)0100100005020117?000103101200(8 9)1001100301111002 \\
\hline 44 & Primulaceae & 00110(6 7)22?00112421113110(0 1 2)0000?(0 3)911(0 1 2)010110(0 1)11110000110005(0 2)(3 C Z)0(0 1)101?1(0 2)0331(4 B)00(0 1)(1 2)1100110030111100(2 3) \\
\hline 45 & Brassicaceae & $\left(\begin{array}{l}0 \\
1\end{array}\right.$ 3)(0 2 3)11(0 1)6?(1 2)?001(0 1)14(0 1)1?11111000(0 1)1?(0 1)(4 5)11(0 2)(B D)10110(0 1)12001050(0 1)(0 1)00(0 1)5020(0 1)1001(2 4)013313100(1 2)A1001120301110002 \\
\hline 46 & Monimiaceae & 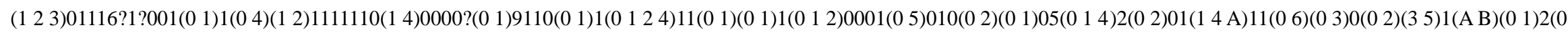 \\
\hline
\end{tabular}


1)1(6 8 9)100110030111110(1 2)

\begin{tabular}{ll}
47 & Basellaceae \\
48 & Piperaceae \\
50 & Lauraceae \\
51 & Gyrostemonaceae \\
Chloranthaceae \\
2 & Phytolaccacae \\
53 & Cucurbitaceae \\
54 & Alismataceae \\
55 & Amaryllidaceae \\
56 & Najadaceae \\
57 & Hydrocharitacee \\
58 & Hamaelidaceae \\
59 & Gomortegaceae \\
60 & Eupomatiaceae \\
61 & Grosssulariaceae \\
62 & Iteaceae \\
63 & Penaeaceae \\
64 & Parnassiaceae \\
65 & Ulmaceae \\
66 & Turneraceae \\
67 & Neuradaceae \\
68 & Nitrariaceae \\
69 & Symplocaceae \\
70 & Portulacaceae \\
71 & Aizoaceae \\
\hline
\end{tabular}

041106?2?00111??1?1?110(0 1)000(0 6)?09112(0 1)1(0 1)11(0 1)111(0 1)101001020(0 1)5(0 2)2001401300331A0402B1001100301110002

$\left(\begin{array}{ll}1 & 2\end{array}\right)(2$ 3)1116?1?00112??1?0?110(0 3)000(0 6)?09110(0 5 B)10110114222000002005020117?0(2 4)00331A02003000110030111100?

(1 2 3)01116?1?001(0 1)241101(0 1)110(0 1 2)000(0 6)?(0 1 4)9110(0 1)1(0 2)11(0 1)(0 1)1500015010(0 2)(0 1)05120014?15(0 3)0431B(0 1)(4 6)0(0 1)(3 8)1001100301110(0 1)0?

(1 3)?1116?1?001(2 3)142111(0 1)110(0 3)00(0 1)0?0(3 5 9)11(0 2)012111(0 1)130000001000502(3 9)0?1???(0 6)00(0 1)30(0 1 2)?201(2 6)1001150301111002

(0 13 3)(0 2)11(0 1)6?1?00110011(0 1)1(0 1)11010010?1911011(0 2)110103000000101(0 1)05(0 1)20(0 1)10005(0 1)0431B?201(7 8 9)1001100301111002

(0 12 3)011(0 1)6?1?001(1 3)24(0 1)1111110(0 3)00(0 1)0?0911(0 2)(0 1)1(0 2)11(0 1)(0 1)(0 1)(1 2)001000(0 1)0001502001601(0 6)01(0 2)31A?202(3 8)100110030111100?

(0 2)211(0 1)6?1?00111??1?1?110(0 3)(0 1)0(0 1)6?(0 3)011(0 2)01(1 2)111(0 1)11110160002(0 1)0502(0 2)01(0 1 4)4?3302313100131001150301110002

04110A22?000?3530?1?11(0 1)0000(0 3 6)40300?(1 2)1(0 1)11(0 1)1150000(1 5 7)010(1 2)00502001(0 4)??(0 3)000(3 5)1(1 2 A)?(3 6)0(0 14$)(069) 1001100301111003$

04110A22?000?0530?1?1100000340310?010110(0 1)15(0 1)(0 1)0070(0 1)0(0 1)0(0 1)5(0 2)2(1 2)(0 2)01(0 4)?330331(1 2)?0(0 1)1(1 3)1001150301111001

04110A22?000?3530?1?110100(0 1)7?(0 1)3104F1(1 2)11101200000000?(0 4)05?2(0 3)014?1500031A?20161001100301111003

04110A22?001?0??0?11110(0 1 2)000(0 36 7)40(3 D)(0 1)0?11(0 1 2)110(0 1)150000(1 2 5)0(0 1)0(1 2)(0 1)0502(0 2)0(0 1)(4 F)?1(0 3)30231(3 A C)?00(0 1)3100115030111(0 1)003

(1 3)21116?1?00100111?11110(0 3)001(0 6)?(0 3)D11(0 1)11(0 1 2)11(0 1)11(1 2)(0 1)0(0 1)(0 1)20000005(0 1 2)20(0 1)(0 1)0004(0 2)02311?101(0 1)1001150301111002

101116?1?00100??1?1111010000?091101101101?3000100000105120?????(3 4)30231B12?181001100301111002

(1 3)(3 5)1116?1?00110?11?1?11000000?09110110110(0 1)14000100000005020?????0203313?0019100112030111(0 1)002

321116?1?10112421111110000(0 1)(0 6)?1011011(0 2)1(0 1)(0 1)(0 1)1(1 2)100100000005020016?04303313?(0 1)0131001120301111002

(1 3)21116?1?001(1 3)00?1?11110(0 3)00(0 1)0?1911?11011011100(0 1)110100015020016?04(0 3)0131(1 2)?(0 1)0101001150301111002

(0 1 3)01116?1?00102??1?1?110(1 4)00(0 1)0?0911(0 2)(0 B)10110012000100100005020?????200331(1 2 A B)?101111011(0 5)0301110002

00110722?00??1????1?110(0 3)0006?0E11?010110(0 1)1100(0 1)050112025020(0 1)1(0 8)012(0 3)03213(0 1)001(0 1)1001120301110002

12111601?001(0 1)142101(0 1)11000010?1911001(0 1 2)11(0 1)113(0 1)(0 1)0100102015020(0 1)1301430330B1201(2 7)1001100301110002

(0 1 3)21116?(1 2)?001024?111(0 1)110000(0 1)0?(0 1)9110(0 B)10110(0 1)1100116001000502001001(2 3)00(2 3)313?001(0 1)1001120301111002

021106?2?001?23?1?1?110000(0 1)(0 6)?(0 1)91100101100111101?010001502001000(0 6)(2 3)0231(1 B)?10(0 1)010011(0 5)0301110002

321116?1?001?23?1110110(0 3)00(0 1)(6 7)?(0 3)5112(0 1 5)101101111001?0100005020016??(0 6)00331(1 B)?20(0 1)(3 8)10011(0 5)0301110002

(1 3)01116?1?00100(1 4)?1111110(0 3)0000?0911011(0 1)110(0 1)111100?01100152(3 9)001011(0 6)(2 3)03311?111(3 8)1001150301111002

(0 3)01106?(1 2)?001(0 1)271101?110(0 1)00(0 1)(0 7)?0(3 5 9)11(0 2)(0 1 C)10110(0 1)11(0 1)(0 1)012010000502001401(3 4)(0 3)033(1 2)(4 A)?00(1 2)(0 1)10011(0 5)0301110002 (0 3)0110622?001?2?11113110(0 1)0000?0911?(1 B)10110(0 1)11000120(0 1)000050200141?6(0 3)0(0 2)3(1 2)2?(0 1)0(1 2)(0 1)1001150301110002 


\begin{tabular}{|c|c|c|}
\hline 72 & Cactaceae & (1 3)01116?1?1011111(0 1)?1?0205200042A003(1 C)10110(0 1)000001(2 6)0100005020014113(0 3)0331(3 A)?002310011(0 2)0301110002 \\
\hline 73 & Onagraceae & 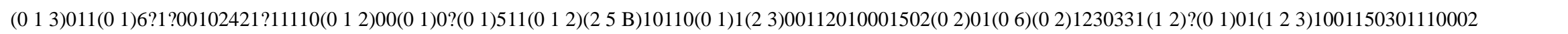 \\
\hline 74 & Trapaceae & 041106?(1 2)?001?1421?011111(0 1)01(0 6)?(0 1)611(0 2)?101100121000?000001502001(0 1)???00331(1 2)?201(2 8)0001150300010002 \\
\hline 75 & Myrtaceae & (1 3)01116???00100???11?110(0 1 2)0000?(0 1)511(1 2)010110(0 1)13(0 1)(0 1)0120(0 1)(0 1)0(0 3 4)15(0 2)20(0 1)1(6 B)206(2 3)0131(1 23$)$ 3)?00(1 2)(0 12$) 10011(25) 0301110002$ \\
\hline 76 & Melastomataceae & (0 2 3)011(0 1)6?(1 2)?001024?111(0 1)11(0 1)10003?(0 1)501(0 2)(0 17 B)1011011(1 2 5)1001601(0 1)0005(0 2)20(0 1)16?1(0 6)(0 3)0331(1 2 A)?00(1 2)(1 3)1001150301110002 \\
\hline 77 & Combretaceae & 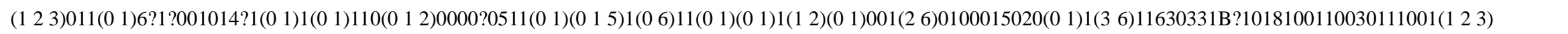 \\
\hline 78 & Poaceae & 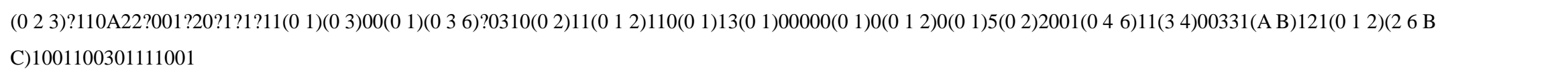 \\
\hline 79 & Orchidaceae & 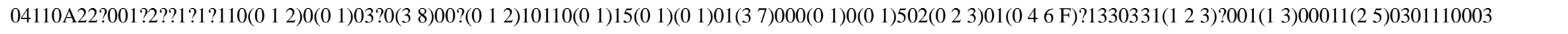 \\
\hline 80 & Araceae & 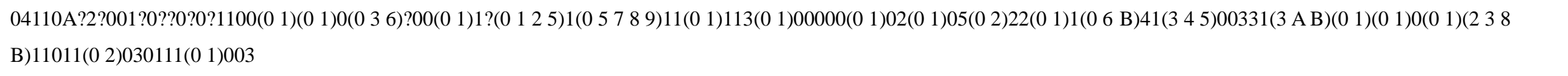 \\
\hline 81 & Cyperaceae & 04110A?2?001?2??1?0?11000003?(0 1)310?11(0 478 9)11(0 1)11300000000(0 1)005?2(0 2)01(4 6)?0(3 4)00(2 3)31(A B)0201(2 68$) 1101100301111003$ \\
\hline 82 & Iridaceae & (0 3)?11(0 1)A?(1 2)?001?1??(0 1)?0?11000(0 1)03?0310?010110(0 1)150001(3 57 8)0(0 1)020(0 1)502(0 2)(0 1)1(l) 4)113(0 3)01312?(0 1)0111001150301111001 \\
\hline 83 & Winteraceae & (1 3)(1 2)1116?1?00003530110110(0 3)0000005110110110(0 1)10(0 1)(0 1)000010(0 1 2)00502(0 2)011000000000000101001120301111002 \\
\hline 84 & Eupteleaceae & (1 3)01116?1?001(0 1)0111111110(0 3)000211911001(0 9)11(0 1)014000000101005(0 1)20013000010200?10121001120301111002 \\
\hline 85 & Papaveraceae & 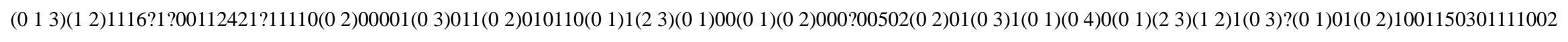 \\
\hline 86 & Ranunculaceae & 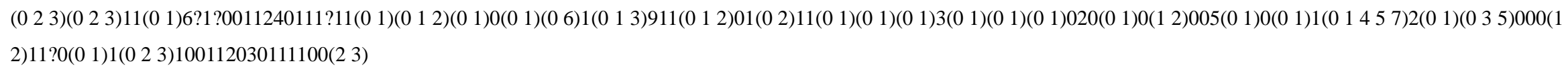 \\
\hline 87 & Sabiaceae & (1 2 3)?11(0 1)6?(1 2)?001(0 1)14011111100000(0 6)009110(0 1)1011011(0 1)00002010(0 2)0050201100(0 1)400311(1 B)131(0 1)(2 3)10011(0 5)0301110002 \\
\hline 88 & Platanaceae & 131116?1?001122111111100001114011?011111101000000101005120(0 1)10000000210?(2 3)00(2 6 9)1001120301111002 \\
\hline 89 & Proteaceae & 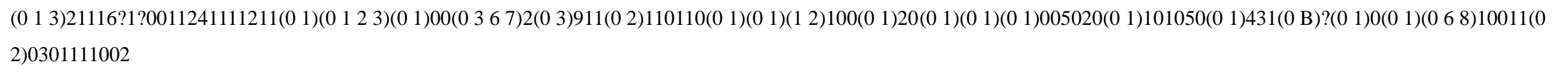 \\
\hline 90 & Trochodendraceae & 1(0 3 5)111601?000(0 1)353011(0 1)1100000(0 1)019110(1 2 4)1(0 4)11(0 1)1(0 1)(0 2)000010101005120(0 1)1100(0 2)201000?001(0 9)1001120301111002 \\
\hline 91 & Buxaceae & (0 1 3)011?6?1?001(0 1 3)020(0 1)111110(0 1)00000(0 1)911051(1 2)111(0 1)(0 1)20000101000(0 1)502001300(2 3)102001?300(1 8)1001150301111002 \\
\hline 92 & Gunneraceae & 04110722?001?10?111?110000(0 1)(0 1)(0 2)(0 1)(0 9 D)01201(1 2)11111300000010(0 2)0050201162?430(2 3)1011201(2 3)1001150301111002 \\
\hline 93 & Myrothamnaceae & $3 ? 1116 ? 1 ? 001002111111101001110911 ? 012111102000000(0$ 1)01005020012?0(2 3)001000?00101001120301111002 \\
\hline
\end{tabular}




\begin{tabular}{|c|c|c|}
\hline 94 & Dilleniaceae & 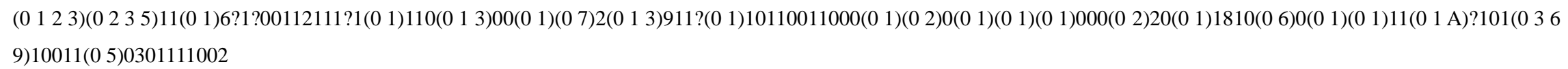 \\
\hline 95 & Erythropalaceae & (1 2 3)?1116?1?001?2(2 4)1111111(0 1)00000(0 3)09110(0 1 5)101101111001200100050231101(0 1)330(2 3)11(A B)(0 1)2113100110030111100? \\
\hline 96 & Olacaceae & 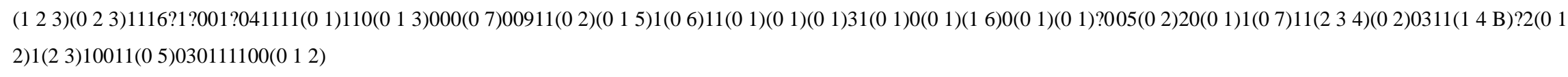 \\
\hline 97 & Aextoxicaceae & $121116 ? 1 ? 001 ? 0(0) 4) 11 ? 10110(0 \quad 12) 000000911 ? 512111111001020100005020116 ? ? 4003111130181001150301111002$ \\
\hline 98 & Berberidaceae & 2(2 3)1116?1?00112(0 4)01?10110(0 3)000(0 6)0(0 1)9110510110(0 1)13000020(0 1)0200512(0 2)(0 1)1(1 3 8)(0 2)0300311(3 A)(0 1)00(0 1)3100112030111100? \\
\hline 99 & Caryophyllaceae & 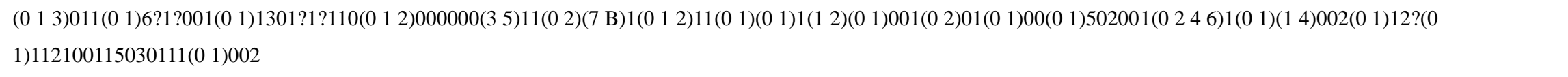 \\
\hline 100 & Nepenthaceae & (0 2 3)?1116?1?001(1 3)140111111(0 1)(0 3)0003?0(3 7)11001211111200003000202502(0 2)11420200211(1 2)000111001150301111002 \\
\hline 101 & Fouquieriaceae & (1 3)01116?1?101?2411?12110000000051120101101110100201(0 1)0015020010003003110?1011100112030111(0 1)002 \\
\hline 102 & Polemoniaceae & 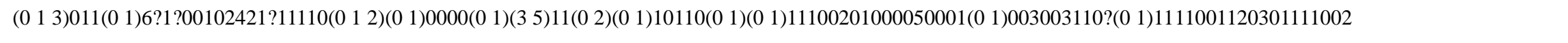 \\
\hline 103 & Theaceae & (1 3)01116?1?00100101110110(0 3)00000(0 1)9110(0 5)10110011(0 1)(0 1)0020(0 1)0(0 1)0(0 1)5020(0 1)14106(0 3)0(2 3)110?00(1 2)01001120301111002 \\
\hline 104 & Actinidiaceae & (1 2 3)(0 2)1116?1?101022(0 1)1?10110(0 3)00(0 1)010(5 9)11001211(0 1)(0 1)1(1 2)100000100?15(0 2)2(0 2)11(6 7)200002110?011(1 4)1001120301111002 \\
\hline 105 & Sarraceniaceae & 04110622?001?0011?1211(0 1)(0 3)000??0G11?010110(0 1)11000020(0 1)000(0 1)50200171?3003110?0(0 1)111001120301111002 \\
\hline 106 & Clethraceae & (1 3)01116?1?00100201110110(0 3)0000019110(0 1 B)1011011111002010(0 2)01522?016203003110?01111001120301111002 \\
\hline 107 & Ericaceae & 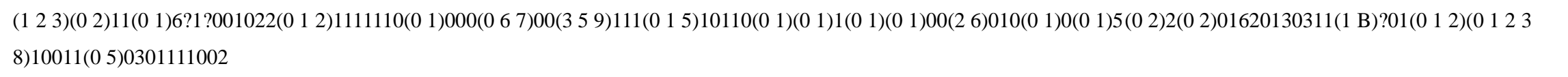 \\
\hline 108 & Cornaceae & (0 1 3)21116?1?001002111101101000130911001011(0 1)1(0 1)210012010001502(0 2)018104303111?211(3 8)1001150301111002 \\
\hline 109 & Alangiaceae & (1 2 3)21116?1?0010(0 2)421?1(0 1)1100000(0 1)30011001(0 2)11(0 1)11(1 2)1(0 1)00201000(0 1)502001(0 3)10(3 4)30311B?2(0 1)131001100301111002 \\
\hline 110 & Nyssaceae & (1 3)21116?1?001?02111101100000030911011(0 1 2)11110100002010(0 1)00500018104303101?211(7 8)1001150301111002 \\
\hline 111 & Loasaceae & (0 1 3)?11(0 1)6?(1 2)?001(0 1)1001?12110(0 1)00012(0 3)(0 4)11?010110(0 1)(0 1)100112010?005030?11103303113?01111001120301111002 \\
\hline 112 & Hydrangeaceae & (0 2 3)?11(0 1)6?1?001(0 1)2011?101101000201911?1101101(0 1)(1 2)100(0 1)20(0 1)00?(0 1)502011000630211(0 3)?11111001120301111002 \\
\hline 113 & Eucommiaceae & 101116?1?00102301111110000000191100121111100000(0 3)010?0050200162(0 1)4003101?31121001150301111002 \\
\hline 114 & Garryaceae & (1 3)21116?1?00111(2 4)0111211010001319110112111112(0 1)00000100005020010004302113?21131001100301111002 \\
\hline 115 & Rubiaceae & 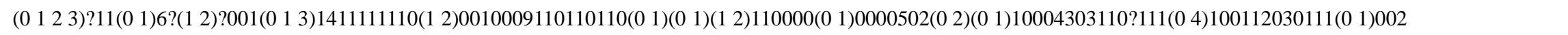 \\
\hline
\end{tabular}




\begin{tabular}{|c|c|c|}
\hline 116 & Loganiaceae & $(0$ 3)(0 2 3)1116?1?001(0 1)1421(0 1)1(1 2)11010010005110(0 1 B)10110(0 1)111100(0 2 6)0100?15020(0 1)1000(3 4)003111?01101001150301111002 \\
\hline 117 & Solanaceae & 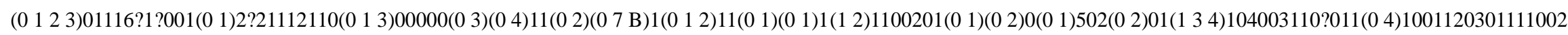 \\
\hline 118 & Taccaceae & 04110A?2?000?3530?1?110(0 3)(0 1)00(0 6)40(5 9)110(0 E)10110115110100000005020011??3303313?01(1 2)(1 3)0001120001111003 \\
\hline 119 & Philydraceae & 04110A?2?000?3530?1?1100010340(3 5)000(0 1 2)1011010510000010(0 1 2)01502(2 3)01F?1300331(1 B)?001(1 3)00011(0 5)0301111001 \\
\hline 120 & Velloziaceae & (0 1)?110A?2?001?2??1?1?1103000341310(0 2)(1 2)10110(0 1)15000110(0 1)0(0 1 2)?(0 1)302(0 2 3)?????330331(2 C)100110001150301111001 \\
\hline 121 & Juncaceae & (0 3)?110A?2?001?2??1?0?11000(0 1)(0 1)340(3 H)00?11(0 2)11(0 1)11500000000(0 1)(0 1)050220?(4 6)?030133(1 2)(2 3 A B)1001(1 46$) 10011(05) 0301111003$ \\
\hline 122 & Scrophulariaceae & $\left(\begin{array}{l}0 \\
1\end{array} 2\right.$ 3)011(0 1)6?(1 2)?00102421?1(0 2)11(0 1)(0 1)00102(1 3)911(0 1 2)(0 7 B)10110(0 1)(0 1)11100(2 6)0(0 1)00(0 1)15(0 2)20010104003110101101001120301111002 \\
\hline 123 & Aquifoliaceae & 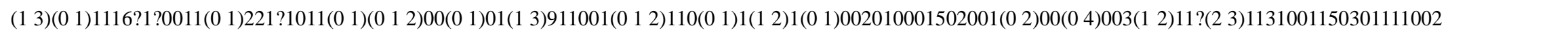 \\
\hline 124 & Campanulaceae & (0 1 3)01116?1?00102421?121100(0 1)000?1911(0 1 2)010110(0 1)(0 1)(1 2)110020(0 1)0000502001(6 8)106303110?01101001120301111002 \\
\hline 125 & Alseuosmiaceae & 3?1116?1?00110(1 4)1101111000000(0 2)151100101101110101201(0 1)0?15020016204303110?11141001120301111002 \\
\hline 126 & Escalloniaceae & (1 3)(0 2)1116?1?001(0 1 3)011111011000000(0 2)(0 1)911?0101101111(0 1)1(0 1)20100015020113104(0 3)0311(0 3)?011(0 3)10011(2 5)030111100? \\
\hline 127 & Bruniaceae & (1 3)?1116?1?0010011111(1 2)1?000003?08??(0 1)010110(0 1)(0 1)1000020100?1502001000430211B?(2 3)11(0 246$) 1001100301111002$ \\
\hline 128 & Araliaceae & (1 3)31116?1?001(0 1)0?21?1111001000?09112B101111(0 1)1(0 1)0(0 1)(0 1)20100(0 5)150200100(0 1)6302111?211(3 4)1001150301111002 \\
\hline 129 & Pittosporaceae & 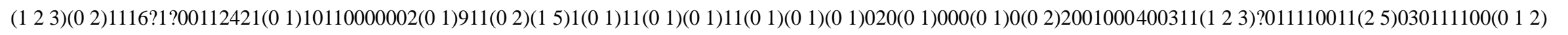 \\
\hline 130 & Adoxaceae & 04110622?00110401?1211011000200110010110(0 1)11110(0 1)20100115?20?10003303110?11131001120301111002 \\
\hline 131 & Caprifoliaceae & 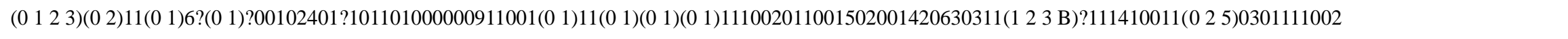 \\
\hline 132 & Saxifragaceae & 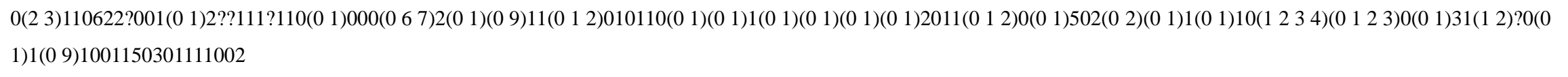 \\
\hline 133 & Crassulaceae & (0 3)(0 2)11(0 1)6?(0 1)?001(0 1)27?1?1?110(1 2 3)(0 1)0002(0 1)9112(B D)10110(0 1)1(1 2)(0 1)(0 1)0120(0 1)0(0 1)005020(0 1)1110(1 2)200110?00101001120301111002 \\
\hline 134 & Penthoraceae & 0011(0 1)62(1 2)?001?0(4 6)111121100000021511?01011011100110010110502011110(0 1)201110?0010100112030111100? \\
\hline 135 & Tetracarpaeaceae & 301116?1?001?2111?1(0 1)110(0 3)0000(0 2)1911?010110112011100001?0502011510(1 2)010110?01101001120301111002 \\
\hline 136 & Altingiaceae & 121116?1?001(0 3)010111(0 1)110000110(0 1)911?11111110000000010?005(0 1)20010004302100?00101001120301111002 \\
\hline 137 & Cercidiphyllaceae & 121116?1?101001111111101001101D1100121111(0 1)?000000101005020013005010200?00101001120301111002 \\
\hline 138 & Hamamelidaceae & (1 3)21116?1?0010010111(0 1)1100001(0 6)0(0 1)911?11(0 1)11(0 1)101001(0 1)201000(0 1)5(0 1)2001000620210(1 2)?201(0 1)1001150301111002 \\
\hline 139 & Geraniaceae & (0 3)211(0 1)6?(1 2)?001(0 1)2321?1?110(0 1)(0 1)01011011(0 2)010110(0 1)10(0 1)010200(0 1)0015020(0 1)1(0 1)(0 1)0100(2 3)100?(2 3)0(1 2)41001120301110002 \\
\hline 140 & Lythraceae & 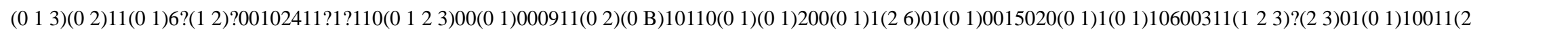 \\
\hline
\end{tabular}




\section{5)0301110002}

141 Staphyleaceae

142 Crossosomataceae

143 Picramniaceae

144 Sapindaceae

145 Tropaeolaceae

Rhamnaceae

Anisophyllaceae

Fagaceae

Betulaceae

Rhoipteleaceae

Myricaceae

Juglandaceae

Celastraceae

Malphighiaceae

Ochnaceae

Rhizophoraceae

Linaceae

Euphorbiaceae

(1 3)21116?1?0010011111(0 1)110(0 1)(0 1)010219110B1(0 1 2)11(0 1)1110000(2 6)010001500(0 1)10103(0 2)0(2 3)110?10101001120301111002

3(0 2)1116?1?00102401112110(0 1 3)00(0 1)0?(0 1)911?01011(0 1)01(1 2)00102010(0 1 2)0(0 2)50200100060101(0 1)0?10(1 24$)(0$ 9)0001120301111002

(1 3)(0 2)1116?1?001?2401?1?1100100000911001211111(1 2)10002010001502011110(3 4)20311(1 2)?(2 3)01(2 3)1001150301110002

(0 12 3)211(0 1)6?(1 2)?00102421?111100100021911?(0 1)1(1 5 6)111(0 1)1(1 2)(0 1)000001000(0 1 2)502(0 2)011103003110?201(0 12347 7)1001120301111002 021106?2?00112(0 4)?1?1?110(0 1)(0 1)0(0 1)61(0 1)D01(0 1)010110013(0 1)0103011100502001(0 1)01300(2 3)1(0 1)1?201(2 3)1001150301110002

(0 12 3)011(0 1)6?(1 2)?0010241111?110(0 1 2)000000911?(0 5)1(0 1 2)11(0 1)(0 1)(0 1)(1 2)11012011000502001(0 3)00(0 14$) 003(012) 1\left(\begin{array}{ll}1 & 2\end{array} 3\right.$ B)?201(3 6 8)10011(2 5)030111(0 1)002

(1 2 3)(0 2)11(0 1)6?(1 2)?001?24(0 1)111(0 1)110(0 1 2)0010009110(0 1)10110113(0 1)00060(0 1)00015020?????330331(1 2)?(0 2)01(1 2)0001150301010002

(0 12 3)(0 23 5)11(0 1)601 ?001(0 1)2411?12110000(0 1)(0 6)21911001(0 1 2)110(0 1)111001(2 6)0(0 1)0(0 1)015(0 2)2(0 2)011101(0 3)0(0 3)11(1 23 A B)?(0 1)(0 1)1(0 6 D)10011(0 $25) 0301110002$

(0 12 3)211(0 1)601?00102421?1(0 1)110(0 1)00(0 1)(0 6)21911(0 2)(0 1 B)10110(0 1)(0 1)(1 2)0011(2 6)010005(0 1)230(0 1)1810(4 5)(2 3)0311(1 2)?2018100115030111(0 1)002

(1 3)01116?1?001(0 13$) 241111(0$ 1)11(0 1)0000(0 6)00911?11011(0 1)(0 1)120010201000150230018102302110?211(2 8)10011203011110(0 1)(2 4)

(1 3)21116?1?001(0 1)2311(0 1)0111000010119110011111(0 1)03(0 1)(0 1)0000100015020(0 1)15104302300?30121001120301110002

(1 3)21116?1?001(0 1)04211011100001011911?011111102000000102005020014214202110?(2 3)112100112030111(0 1)002

041116?1?001?14111111100101021511?01(0 7)10(0 1)10200005010?005020014214002102120(1 2)21001150301110002

(1 3)(0 2)1116?1?00112211111110000001(0 1)511?01(1 2)111103000(0 1)00(0 1)02005020(0 1)14214302102?41031001150301110002

1(2 5)1116?1?0010141111(0 1)110(0 1)00(0 1)02(0 1)511001(1 2)11110200000010?(0 1)05020014214302102?41021001150301110002

(1 2 3)011(0 1)6?1?001(0 1)24(0 1)111?110(0 1)00(0 1)001911(0 2)(0 125 B)1(0 1 2)11(0 1)11(1 2)100020(0 1)0(0 2)(0 1)0502(0 2)010006(0 2)0311(1 2)?(2 3)01(1 367

8)1001150301111002

(1 2 3)(0 2)1116?1?00102421?1(0 1)110100(0 1)00(0 1)911(0 2)(0 1)1(0 5)11(0 1)111(0 1)01000000015(0 2)20010(1 2)0300(0 2)11(0 12 B)?201(2 4789$) 10011(025) 0301110002$

(1 3)(2 3)1116?1?00102411?10110(0 3)00000(0 1)911?(0 1)1011011(1 2)00(0 1)000(0 1)01005(0 2)2300(6 7)10(0 6)003513?(0 1)010100112030111(0 1)002

(0 3)211(0 1)6?(1 2)?0010241111(0 1)110(0 1)00(0 1)0?0(5 9)11011011011(1 2)(0 1)0(0 1)0200(0 1)00(0 1)502001(4 7)106003111?301(0 8)1001150301111002

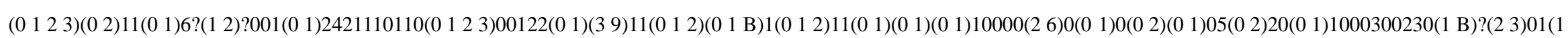
$248) 10011(05) 0301111002$ 
Table S2. Characters (The numbers in the parentheses are the values of each characters).

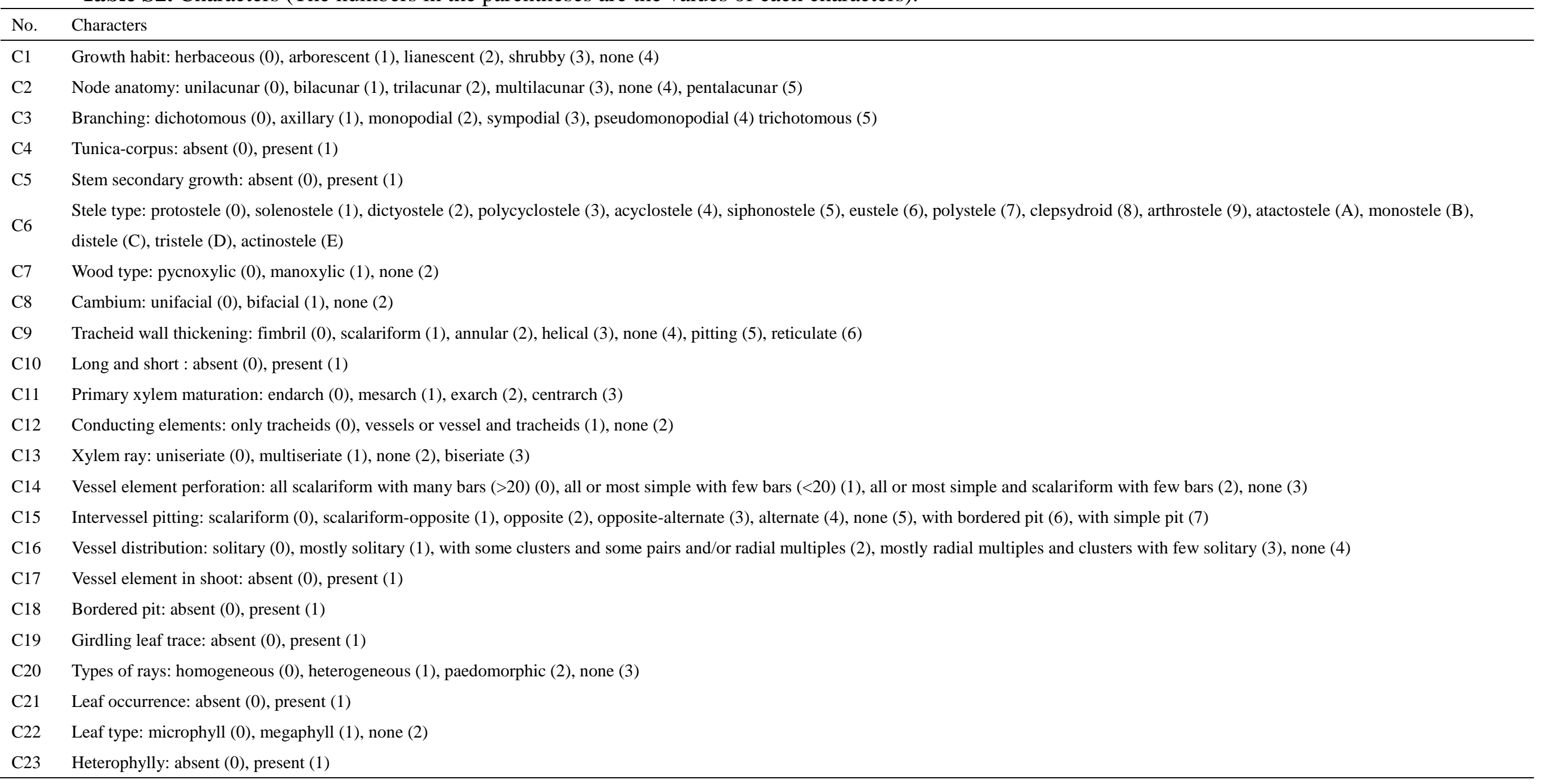


Leaf organization: simple (0), compound (1), none (2)

Ensiform lamina: absent (0), present (1)

Stipule: absent (0), present (1)

Venation: pinnate (0), actinodromous pinnate but with basal concentration of laterals parallel (1), dichotomous other palmate (2), one-veined (3), none (4)

C29 Secondary veins: brochidodromous (0), craspedodromous (1), semicraspedocromous (2), camptodromous (3), none (4)

C30 Leaf margin: smooth (0), serrate (1), none (2), dissected lobate (3),

Leaf/pinna shape: lobate (0), lingular (1), wedge-shaped (2), linear (3), dissected lanceolate (4), fan-like (5), strap-like (6), scale-like (7), oboval (8), none (9), needle-like(A), bifid (B), orbicular (C),

cordate-based (D), peltate (E), tubular $(\mathrm{F})$, teret $(\mathrm{G})$

C32 Midrib: absent (0), present (1)

C33 Vein reticulation: absent (0), present (1)

C34 Stomata occurrence: hypostomatic (0), hyperstomatic (1), amphistomatic (2), on branch (3), none (4), on sporangia (5)

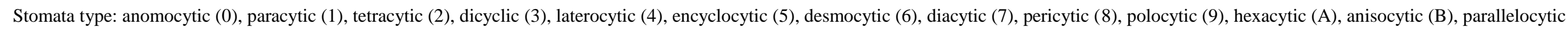

(C), heliocytic (D), axillocytic (E), none (F)

Heterospory: absent (0), present (1)

Gender: bisexual (0), monoecious (1), dioecious (2), none (3), androdioecious (4), polygamomonoecious (5), polygamodioecious (6), gynomonoecious (7), gynodioecious (8), andromonoecious (9)

C38 Siphonogamy:absent (0), present (1)

C39 Reproductive and vegetaive organ : intermixing (0), separated (1), none (2),

C40 Mega- and microsporangia arrangement: proximal (0), segregated (1), none (2),

C41 Reproductive parts concentrated to the distal: absent (0), present (1)

C42 Reproductive organ (flower/cone) base: sessile (0), pedicellate (1), none (2)

C43 Reproductive organ peripheral foliar appendage merism: numerous and helical (0), pentamerous (1), tetramerous (2), few and variable (3), none (4), trimerous (5), hexamerous (6)

C44 Reproductive organ 1st cycle peripheral foliar appendage connation: absent (0), present (1), none (2)

C45 Reproductive organ 2nd cycle peripheral foliar appendage connation: absent (0), present (1), none (2)

C46 Petal clawed: absent (0), present (1), none (2)

C47 Hypanthium:absent (0), present (1) 
C48 Nectary : absent (0), abaxial (1), gynoecial (2), surface (3), disc peripheral (4), foliar (5), appendage other position and roecial (6), extrafloral (7), perigonal (8), stylar (9), base stigmatic (A)

C49 Microsporangia grouping: synangiate (0), distinct (1)

C50 microsporangium arrangement: connate (0), free (1), none (2)

C51 Heterostyly: absent (0), present (1), none (2)

C52 Anther orientation: introrse (0), latrorse (1), extrorse (2), none (3)

C53 Anther type: tetrasporangiate (0), bisporangiate (1), none (2), trisporangiate (3), unisporangiate (4), multisporagniate (5)

C54 Microsporangium insertion: basifixed (0), dorsifixed (1), ventrifixed (2)

C55 Microsporangia position relative to support structure: absent adaxial (0), abaxial (1), marginal (2), helical (3), terminal (4), peripheral (5), between bracts (6)

Microsporangia opening: longitudinal slit (0), valve pore (1), none (2), flap oblique slit (3), horizontal slit (4), apical slit (5), helical slit (6), decay radial slit (7)

C57 Saccate pollen: bisaccate (0), monosaccate (1), none (2), pseudosaccate (3), tetrasaccate (4), trisaccate (5),

C58 Microspore grouping: monad (0), dyad (1), tetrad (2), polyad (3)

C59 Microspore size: $>20$ um (0), < 20 um (1)

C60 Tectum: absent (0), present (1)

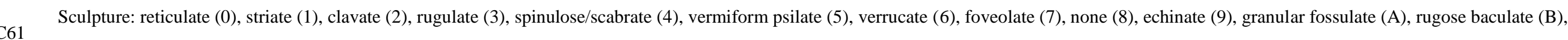
gemmate (C)

C62 Tectal perforations: semitectate (0), tectate (1), perforate (2), imperforate (3), none (4), atectate (5),

C63 Columellae :absent (0), present (1)

C64 Number of ovule-enclosing unit (carpel/cupule) per reproductive organ: >5 (0), 5 (1), 4 (2), 2 (3), 1 (4), 1 -5 (5), none (6),

C65 Gynoecium positioning: superiort (0), only extreme baset (1), inferior basalt (2), half inferiort (3), mostly to completely inferiort (4), nonet (5)

C66 Ovule-enclosing unit (carpel) stipitation: absent (0), present (1)

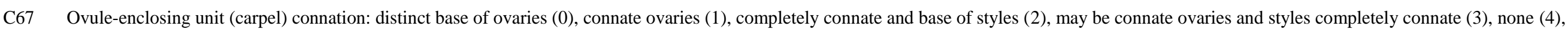

C68 Ovule-enclosing unit distal projection (style) position: eccentric (0), peak of ovary (1), intercalated to each other (2), absent terminal (3), decurrent (4), gynobasic (5)

C69 Non-nucellar pollen receiving part (stigma) position: decurrent (0), ventral (1), apical (2), branched (3), none (4)

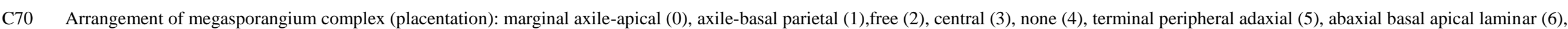

C71 Pendulous ovules: absent (0), present (1)

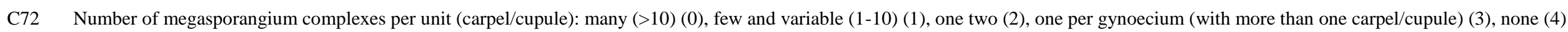


C73 Number of megasporangia surrounding layers (integument): two (0), one (1), none (2)

C74 Megasporangium complex orientation: orthotropous (0), anatropous (1), campylotropous (2), none amphitropous inverted (3),

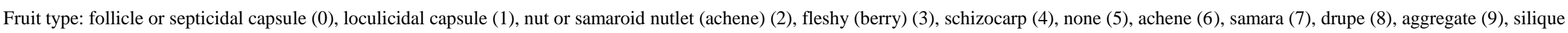
(A), utricle (B), caryopsis (C), pome (D)

C76 Spermatozoid: absent (0), present (1)

C77 Number of functional megapsores per megasporangium: one (0), more none (1)

C78 Megaspore tetrad: linear (0), tetrahedral (1), none (2),

C79 Megasporangium complex enclosed: absent (0), present (1)

C80 Megasporangia retained in on mother plant: absent (0), present (1)

C81 Megasporangium position relative to support structure: terminal abaxial (0), adaxial marginal lateral and isolated peripheral (1), none (2)

C82 Ovule-wrapping unit (cupule): absent (0), present (1)

C83 Ovule-wrapping unit (cupule) opening: distal adaxial (0), proximal abaxial (1), proximal none adaxial (2),

C84 Micropylar tube: absent (0), present (1)

C85 Pollination through pollen tube:absent (0), present (1)

C86 Archegonia in megaspore: absent (0), present (1), none (2)

C87 Double fertilization: absent (0), present (1)

C88 Endosperm: absent (0), present (1)

C89 Angiocarpy: absent (0), present (1)

C90 Vivipary: absent (0), present (1)

C91 Number of cotyledon: many (0), 4-3 (1), 1 (2), none (3) 
Table S3. Taxa studied and GenBank accessions. A dash (-) indicates missing data, and the indicated sequences are from GenBank.

\begin{tabular}{|c|c|c|c|c|c|}
\hline & matK & $r b c L$ & ITS & $\operatorname{trnL}$ & psbA-trnH \\
\hline Actinidiaceae & KP737312 & KP737327 & KP737354 & JN102166 & KP737342 \\
\hline Adoxaceae & EF490235 & KM360613 & U88194 & AF366927 & FJ395478 \\
\hline Aextoxicaceae & DQ182342 & HE651123 & AY590831 & AY145362 & - \\
\hline Aizoaceae & JQ844150 & AJ235778 & AJ582944 & KF132800 & KF132892 \\
\hline Alangiaceae & FJ644649 & DQ340449 & KP092580 & JN102141 & JF321228 \\
\hline Alismataceae & AB040179 & HQ901563 & DQ339086 & GQ244545 & KC584951 \\
\hline Alseuosmiaceae & AJ429378 & X87377 & EU331124 & AJ430965 & - \\
\hline Altingiaceae & AF015650 & JX944788 & GU576669 & DQ352220 & KP095965 \\
\hline Amaryllidaceae & JX464550 & JX903147 & AY280349 & JX464362 & KC704237 \\
\hline Amborellaceae & DQ185522 & L12628 & - & AY145324 & - \\
\hline Aquifoliaceae & AF542607 & KX897006 & AH007153 & FR849986 & KU198261 \\
\hline Araceae & AY034184 & КМ360996 & KJ400913 & AM933296 & KC584957 \\
\hline Araliaceae & AB087999 & U50250 & KF727964 & AF366930 & KX347019 \\
\hline Araucariaceae & AM920149 & U96471 & - & - & FJ17352 \\
\hline Basellaceae & JQ844148 & MF135428 & KY968898 & - & MF143716 \\
\hline Berberidaceae & JQ172882 & KR075627 & KC575606 & FJ626558 & JQ172934 \\
\hline Betulaceae & AY372021 & KC482102 & - & DQ860529 & KP095389 \\
\hline Brassicaceae & AB354274 & AY167979 & GQ202247 & AF451575 & GQ184349 \\
\hline Brunelliaceae & EF135512 & FJ707536 & AY935891 & AF299181 & KJ426624 \\
\hline Bruniaceae & AY490949 & L14391 & AY494012 & GQ984070 & - \\
\hline Buxaceae & LN877447 & MF349741 & AF245412 & LN877711 & AB331298 \\
\hline Cactaceae & HМ041657 & KT164772 & AY181566 & НМ041236 & KT222807 \\
\hline Campanulaceae & EU713264 & AJ419698 & KC013706 & GQ984060 & - \\
\hline Caprifoliaceae & GQ997392 & GQ997430 & JQ780992 & HM228560 & GU135313 \\
\hline Caryophyllaceae & KU722867 & M77699 & KU722881 & JN589634 & GU070803 \\
\hline Celastraceae & EU002170 & EU002277 & EU002149 & HQ393763 & GQ998178 \\
\hline Cephalotaxaceae & AF457108 & AF456387 & JF976114 & EF660619 & EF660701 \\
\hline Cercidiphyllaceae & AB490203 & L11673 & AF147756 & AB445242 & AB445234 \\
\hline Chloranthaceae & AF543733 & AF332097 & D29787 & AY145334 & KP159335 \\
\hline Clethraceae & MF350258 & L12609 & KU350161 & AJ430871 & MF348393 \\
\hline Combretaceae & GU135057 & FJ381811 & KT279737 & LC054306 & KP319003 \\
\hline Connaraceae & EU002174 & L29493 & AY935892 & KC428477 & KX248193 \\
\hline Cornaceae & EU002175 & EU002276 & AY152522 & AY254238 & JF321206 \\
\hline Crassulaceae & AF115647 & JX848453 & AB088618 & KX510105 & KP159333 \\
\hline Crossosomataceae & DQ443456 & DQ443456 & DQ307116 & DQ307148 & KX999391 \\
\hline Cucurbitaceae & DQ536662 & L21937 & КС922448 & HM597017 & JQ007373 \\
\hline \multirow[t]{2}{*}{ Cunoniaceae } & JX517913 & JX572494 & AF521290 & AF299156 & - \\
\hline & & 26 & & & \\
\hline
\end{tabular}




\begin{tabular}{|c|c|c|c|c|c|}
\hline Cupressaceae & AB023999 & L25756 & AY916904 & JF514857 & JF514865 \\
\hline Cycadaceae & AF143440 & AB917042 & KC508028 & HG316537 & KP117157 \\
\hline Cyclanthaceae & KT205206 & KT205272 & JN016949 & KJ681523 & KT205225 \\
\hline Cyperaceae & KX036931 & AM999811 & AF168838 & AJ295759 & KR735884 \\
\hline Davidsoniaceae & AY935930 & AF206759 & - & KC428488 & KM895252 \\
\hline Dilleniaceae & GQ997143 & FJ860350 & AY096030 & KF953925 & KT452064 \\
\hline Encephalartos & GQ203845 & AF394361 & KX130213 & - & KX152014 \\
\hline Ephedra & KP788852 & FJ958029 & FJ958007 & КT033390 & - \\
\hline Ericaceae & U61316 & L12625 & AF382730 & AF271699 & MF348522 \\
\hline Erythropalaceae & DQ790197 & DQ790161 & DQ790230 & EF464487 & - \\
\hline Escalloniaceae & AJ429365 & AJ419694 & U42544 & AJ430952 & KC355696 \\
\hline Eucommiaceae & AF345323 & L01917 & HQ384682 & - & KX346918 \\
\hline Eucryphiaceae & EU002176 & L01918 & - & KF594405 & KM895039 \\
\hline Euphorbiaceae & LK021494 & AY794915 & AY918198 & AY794734 & GU135374 \\
\hline Eupomatiaceae & DQ401341 & DQ861790 & AF469771 & 1842 & 64673 \\
\hline Eupteleaceae & DQ401348 & KR232440 & KR011731 & LN610908 & KR064716 \\
\hline Fabaceae & AF203597 & KM025243 & AF156675 & AY 651848 & GU396719 \\
\hline Fagaceae & FJ185050 & KF418893 & - & AF344182 & - \\
\hline Fouquieriaceae & EU628508 & AY725861 & АH007888 & AJ430876 & JF321292 \\
\hline Garryaceae & KT738340 & KT740821 & JN234737 & JN234724 & KT739195 \\
\hline Geraniaceae & KJ916373 & KJ916495 & DQ525074 & AJ884716 & KP963310 \\
\hline Ginkgo & AF279806 & JQ512538 & JQ279501 & AY145323 & AF223226 \\
\hline Gnetum & AY449621 & U72819 & AY449558 & AY296493 & AY849369 \\
\hline Gomortegaceae & - & AF206773 & AF289846 & - & AF129053 \\
\hline Gunneraceae & EU002179 & EU002279 & AF517101 & AM397168 & GQ998323 \\
\hline Gyrostemonaceae & FJ212199 & L22440 & AF070971 & FJ212281 & - \\
\hline Hamaelidaceae & AF013046 & KC737393 & AF094551 & DQ352357 & KC737342 \\
\hline Hamamelidaceae & AF248617 & AY263940 & AF015654 & GU576826 & GU576755 \\
\hline Hydatellaceae & JQ284136 & JQ284248 & JQ284173 & - & - \\
\hline Hydrangeaceae & GU217324 & JF308660 & GU983035 & JN226776 & GU217324 \\
\hline Hydrocharitaceae & AB088781 & AB088810 & JN578091 & - & - \\
\hline Illiciaceae & DQ185524 & DQ182334 & AF163734 & AY145325 & - \\
\hline Iridaceae & KC118929 & KC704850 & DQ277636 & EU939496 & KC704317 \\
\hline Iteaceae & EF456732 & L11188 & AY231368 & - & AY138110 \\
\hline Juglandaceae & HE966942 & KF418920 & HM049898 & AY231167 & HE966671 \\
\hline Juncaceae & AY973527 & L12681 & KR082780 & AY344156 & HQ181837 \\
\hline Lauraceae & KU564571 & KU564795 & HG315591 & KX674308 & KU564671 \\
\hline Linaceae & JX661951 & FJ169596 & KX147539 & FJ160887 & GQ845215 \\
\hline Loasaceae & KY286710 & U17876 & KY286627 & KY286979 & JF321277 \\
\hline Loganiaceae & KJ815653 & Z68826 & DQ358879 & AJ430910 & - \\
\hline
\end{tabular}




\begin{tabular}{|c|c|c|c|c|c|}
\hline Lythraceae & GU228449 & L10223 & - & AY905482 & JQ730675 \\
\hline Magnoliaceae & AY00900 & AY008933 & EU593549 & AY305819 & HM236892 \\
\hline Melastomataceae & KP093302 & GQ436728 & KY798014 & GQ265885 & JF708230 \\
\hline Monimiaceae & AJ247183 & AF040664 & GU177689 & AF129041 & AF129068 \\
\hline Myricaceae & KF419021 & KF418924 & KP092762 & DQ501450 & KP095418 \\
\hline Myrothamnaceae & AM396507 & AF060707 & AF094555 & AM397169 & EU213839 \\
\hline Myrtaceae & AF368206 & AB537496 & - & HQ287716 & KP142183 \\
\hline Najadaceae & HM240463 & U03731 & KT596618 & KT596674 & AB331276 \\
\hline Nepenthaceae & AF315891 & L01936 & AB675865 & JX042575 & MF348377 \\
\hline Neuradaceae & - & U06814 & KJ004316 & - & - \\
\hline Nitrariaceae & - & DQ267158 & DQ267176 & DQ267166 & КР087763 \\
\hline Nymphaeaceae & DQ185527 & LC219121 & JF805747 & AF543729 & KC525065 \\
\hline Nyssaceae & JF308675 & JF308651 & EU734443 & EU734496 & JF321237 \\
\hline Ochnaceae & KF263283 & KF263405 & KF263218 & HQ599792 & - \\
\hline Olacaceae & GQ997871 & GQ997898 & DQ333869 & DQ340620 & GQ997883 \\
\hline Onagraceae & HM851006 & EU255778 & GU176556 & GU176588 & - \\
\hline Orchidaceae & AF263677 & AF074211 & AY273748 & AY273651 & FJ460374 \\
\hline Oxalidaceae & EU002186 & EU002282 & EU436875 & JN639556 & GQ998541 \\
\hline Papaveraceae & KM364817 & U86629 & - & AY328253 & - \\
\hline Parnassiaceae & JF954971 & JN105081 & JF811045 & DQ860575 & JF802315 \\
\hline Penaeaceae & AY151582 & AY151706 & AM235864 & - & AY151675 \\
\hline Penthoraceae & KC988293 & AF190431 & KC988286 & X71987 & DQ006173 \\
\hline Philydraceae & EU499296 & AY298834 & - & AJ387741 & - \\
\hline Phytolaccaceae & AY042631 & FJ860398 & KU377334 & KM261955 & MF143636 \\
\hline Picramniaceae & - & AF127025 & - & - & KJ426870 \\
\hline Pinaceae & EF440492 & EF440570 & - & $\mathrm{EF} 440530$ & FJ493294 \\
\hline Piperaceae & DQ882215 & EF591363 & KF924100 & EU519779 & KM055199 \\
\hline Pittosporaceae & DQ133794 & HM850262 & HM116994 & AJ430960 & KM895297 \\
\hline Platanaceae & EU642711 & AF081073 & AY706029 & AY145358 & KP402512 \\
\hline Poaceae & KM487287 & AY836162 & - & JX456355 & HQ894425 \\
\hline Podocarpaceae & AF228112 & AF249648 & AB023989 & EF660630 & JN041002 \\
\hline Polemoniaceae & L34205 & KX397893 & AF167227 & EF433257 & KJ686557 \\
\hline Portulacaceae & EU834751 & M62568 & JF508549 & - & MF143649 \\
\hline Primulaceae & HQ535964 & HQ619773 & - & KM198463 & - \\
\hline Proteaceae & AY823204 & JX571862 & - & AF482140 & - \\
\hline Ranunculaceae & EU827661 & DQ099444 & FJ424225 & KP900425 & KY235737 \\
\hline Rhamnaceae & GQ434248 & KP121449 & DQ146573 & KR083150 & HG765028 \\
\hline Rhizophoraceae & JX661962 & JX664070 & AF130332 & AY947404 & JX663300 \\
\hline Rhoipteleaceae & U92852 & AF017687 & AF303800 & AY147081 & KF201474 \\
\hline Rosaceae & DQ860466 & JQ391365 & JQ392451 & DQ863238 & JQ390723 \\
\hline
\end{tabular}




\begin{tabular}{llllll} 
Rubiaceae & KJ815700 & FJ493349 & - & KJ815420 & MF348541 \\
Sabiaceae & HM755905 & HM755932 & KM092335 & KP900465 & HM755920 \\
Sapindaceae & KP675825 & FN599461 & KP675785 & JN102157 & KJ687156 \\
Sarraceniaceae & JQ619005 & KR819588 & DQ098117 & AJ430886 & MF348369 \\
Saxifragaceae & KC737243 & KC737395 & KC004032 & JN102287 & JF708213 \\
Scrophulariaceae & KR424749 & EF544598 & EF363673 & DQ856494 & KR424763 \\
Solanaceae & EF537313 & AF397102 & KP006659 & EU603443 & EF537224 \\
Staphyleaceae & EU002189 & EU002285 & KR349376 & KX999498 & KX999401 \\
Symplocaceae & AF380108 & AY725865 & MF171080 & AF534682 & EU769795 \\
Taccaceae & AY973837 & JF944589 & JF978861 & JN850578 & JN047324 \\
Taxodiaceae & AB030115 & JQ512531 & AB023983 & AY727216 & JQ512286 \\
Tetracarpaeaceae & L34154 & L11207 & EF178790 & - & - \\
Theaceae & - & AF380058 & MF171105 & HM100596 & HM100528 \\
Thymelaeaceae & KU244186 & GQ436619 & KX024773 & GU736358 & KU244056 \\
Trapaceae & KX526694 & L10226 & FM887019 & AY905491 & - \\
Trochodendraceae & AF543751 & GQ998840 & AF192799 & AY145360 & GQ998829 \\
Tropaeolaceae & FM179931 & L14706 & JN115053 & AB043665 & - \\
Turneraceae & JX661965 & DQ123398 & DQ521285 & JQ723403 & JX663303 \\
Ulmaceae & KC539623 & KC539693 & KC539589 & KC539738 & - \\
Velloziaceae & JX286710 & AY149358 & JN016957 & AF293095 & JN017061 \\
Vochysiaceae & GQ982128 & AM235665 & DQ787415 & - & KX249560 \\
Welwitschia & AF280996 & AF394335 & U50740 & AY513733 & AY849370 \\
Winteraceae & EU669474 & EU669518 & AY526318 & EU669556 & FJ539202 \\
Zamia & GQ203859 & JQ770263 & AJ287364 & - & JQ770199 \\
\hline & & & & &
\end{tabular}




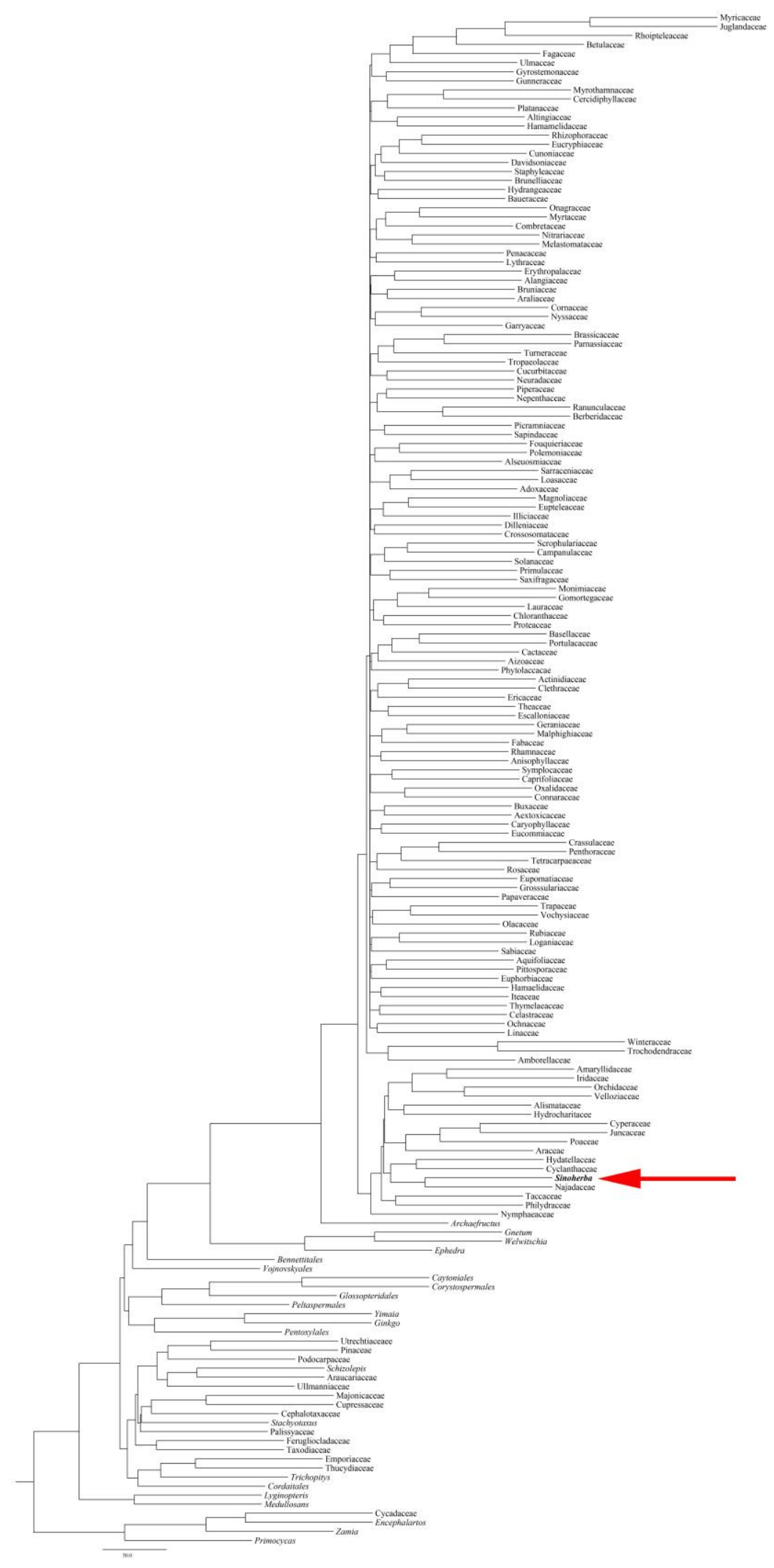

Figure S1. Phylogenetic relationships of the morphological characteristic score information. The numbers near the nodes are bootstrap percentages. "**" indicates that the node is $100 \%$ supported. "६" indicates the phylogenetic position of Sinoherba. 


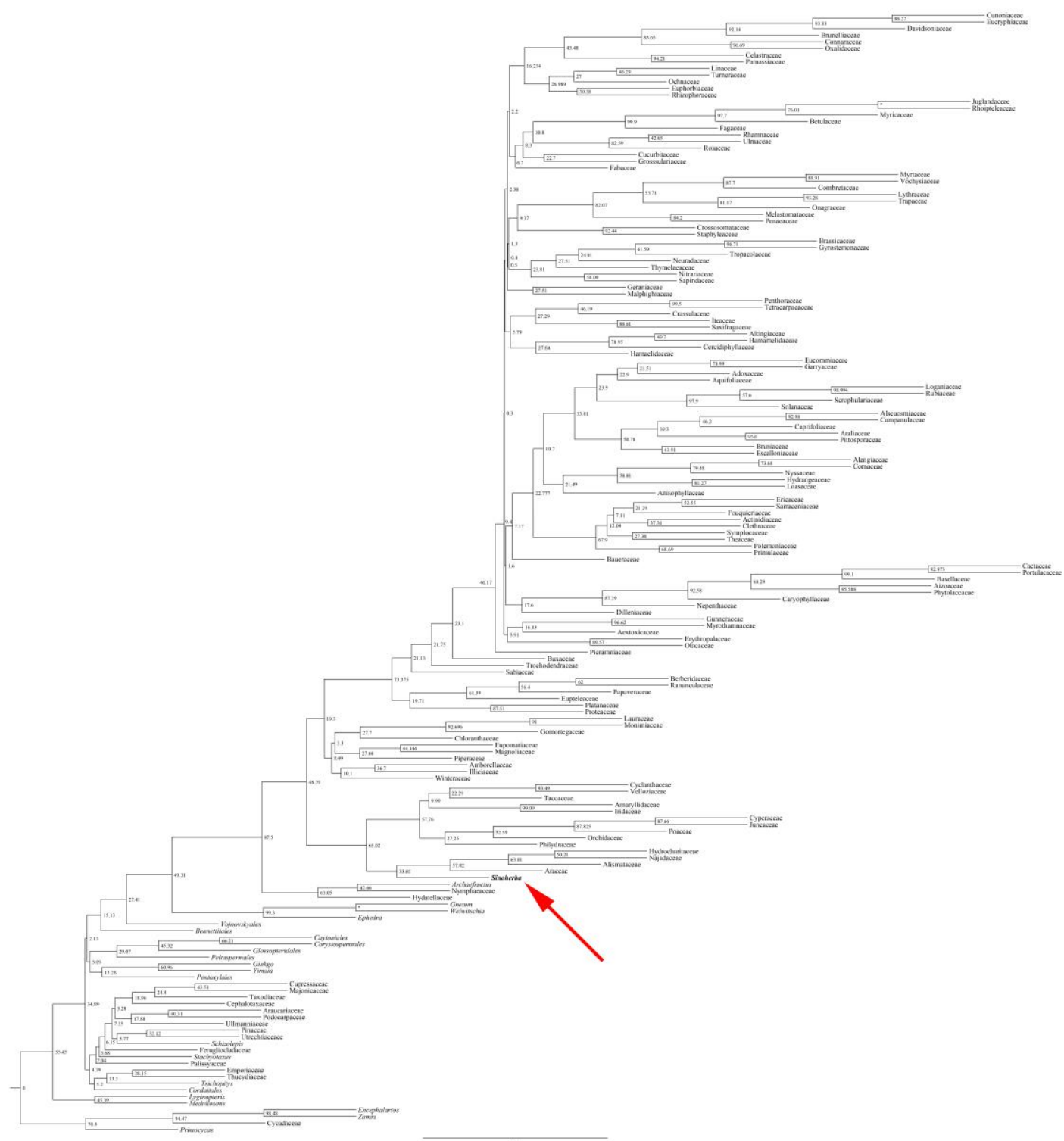

Figure S2. Phylogenetic relationships based on DNA sequence ( $r b c L$, matK, $\operatorname{trnL}-F$, psbA-trnH and ITS) and morphological characteristic score information of Sinoherba. The numbers near the nodes are bootstrap percentages. "*" indicates that the node is $100 \%$ supported. "«" indicates the phylogenetic position of Sinoherba. 